\title{
High frequency VLBI observations of the scatter-broadened quasar B 2005+403 $\star$
}

\author{
K. É. Gabányi ${ }^{1}$, T. P. Krichbaum ${ }^{1}$, S. Britzen ${ }^{1}$, U. Bach ${ }^{1,2}$, E. Ros $^{1}$, A. Witzel ${ }^{1}$, and J. A. Zensus ${ }^{1}$ \\ 1 Max-Planck-Institut für Radioastronomie (MPIfR), Auf dem Hügel 69, 53121 Bonn, Germany \\ e-mail: gabanyik@mpifr-bonn.mpg.de \\ 2 Istituto Nazionale Di Astrofisica (INAF), Osservatorio Astronomico di Torino, via Osservatorio 20, 10025 Pino Torinese, Italy
}

Received 9 August 2005 / Accepted 16 January 2006

\begin{abstract}
The quasar B 2005+403 located behind the Cygnus region in our Galaxy, is useful in studying the interplay between propagation effects, which are extrinsic to the source, and source intrinsic variability. On the basis of VLBI experiments performed at 1.6, 5, 8, 15, 22, and 43 GHz during the years 1992-2003 and parallel multi-frequency monitoring of the total flux density (between 5-37 GHz), we investigated the variability of total flux density and source structure. Below $8 \mathrm{GHz}$, the point-like VLBI source is affected by scatter-broadening of the turbulent interstellar medium (ISM), which is located along the line of sight and likely associated with the Cygnus region. We present and discuss the measured frequency dependence of the source size, which shows a power-law with slope of $-1.91 \pm 0.05$. From the measured scattering angle at $1 \mathrm{GHz}$ of $\theta=77.1 \pm 4.0$ mas a scattering measure of $\mathrm{SM}=0.43 \pm 0.04 \mathrm{~m}^{-20 / 3} \mathrm{kpc}$ is derived, consistent with the general properties of the ISM in this direction. The decreasing effect of angular broadening towards higher frequencies allows us to study the internal structure of the source. Above $8 \mathrm{GHz}$ new VLBI observations reveal a one-sided, slightly south-bending core-jet structure, with stationary and apparent superluminallymoving jet components. The observed velocities range from 6.3-16.8 c. The jet components move on non-ballistic trajectories. In AGN, total flux density variations are often related to the emergence of new VLBI components. However, almost eleven years no new component was ejected in B 2005+403. A striking feature in the flux density variability is a trough observed at 5-37 GHz and between 1996 and 2001 . This trough is more pronounced at higher frequencies, where it shows a prominent flux density decrease of $\sim 50-60 \%$ on a time scale of $2-3$ yr. The trough can be explained as a blending effect of decreasing and increasing jet component fluxes. Densely time-sampled flux density monitoring observations with the $100 \mathrm{~m}$ Effelsberg telescope reveal an intra-day variability (IDV) at $1.6 \mathrm{GHz}$ with a modulation index of $m=1.0 \%$. At $5 \mathrm{GHz}$ less pronounced variations are seen $(m=0.5 \%)$. This and a relatively short variability time scale of $\sim 0.1$ days imply a second, less dense or turbulent scattering screen at nearby (few to hundred parsec) distance.
\end{abstract}

Key words. galaxies: jets - galaxies: active - galaxies: quasars: individual: B 2005+403

\section{Introduction}

The propagation of radio waves through the ionized interstellar medium causes several effects, such as Faraday rotation and depolarization of polarized emission, dispersion of pulsar signals, scatter-broadening of compact radio sources and intensity fluctuations caused by diffractive (DISS) and refractive (RISS) interstellar scintillation (ISS). The effect of scatter broadening plays an important role in our understanding of the compact object located in the center of our galaxy Sgr A* (e.g. Doeleman et al. 2001; Krichbaum et al. 1998b; Lo et al. 1998). It is however also important for other galactic regions on the sky, e.g. for the Cygnus region and the quasar B 2005+403 located behind it. The detailed study of scatter broadening in compact radio sources could lead to a better understanding of the interstellar medium. It also has the potential to reveal fine details of the

* Table 2 is only available in electronic form at http://www. edpsciences.org intrinsic source structure, particularly at longer wavelengths, where direct imaging often cannot provide sufficiently high angular resolution.

The other prominent propagation effect addressed in this paper is the so-called Intra-Day Variability (IDV, Witzel et al. 1986; Heeschen et al. 1987), and the question of if and how it is related to the interstellar scintillation. Recent IDV surveys show that a large fraction (up to $30 \%$ ) of all compact flat spectrum radio sources show this effect, which is characterized by variability amplitudes of up to $20-30 \%$ and variability timescales ranging from less than one hour to several days (e.g. Kraus et al. 2003; Heeschen et al. 1987; Kedziora-Chudczer et al. 2001; Lovell et al. 2003). If interpreted via source intrinsic incoherent emission processes, such short variability timescales imply - via the light travel time argument - apparent brightness temperatures of $T_{\mathrm{B}}=10^{16 \ldots 19} \mathrm{~K}$, far in excess of the inverse-Compton limit (Kellermann \& Pauliny-Toth 1969). With the assumption of relativistic Doppler-boosting 
the brightness temperatures can be reduced, which however would imply uncomfortably large Doppler boosting factors of $\delta \simeq 20-200$.

Alternately, the IDV in the radio-bands is also explained extrinsically via scintillation of radio waves in the turbulent interstellar medium (ISM) of our Galaxy (e.g. Rickett 1990; Dennet-Thorpe \& de Bruyn 2000). The main problem in this interpretation is that it lacks the explanation of observed radio-optical broad-band correlations in at least some sources (Quirrenbach et al. 1991, see also Wagner \& Witzel 1995). The recent detection of diffractive ISS in the source J $1819+3845$ leads to micro-arcsecond source sizes and brightness temperatures of $\sim 10^{14} \mathrm{~K}$, which again requires Doppler boosting factors of $\delta \simeq 100$ (Macquart \& de Bruyn 2005). It is therefore likely that the IDV phenomenon invokes both, a combination of source intrinsic and extrinsic effects (e.g. Krichbaum et al. 2002; Qian et al. 2002).

In this paper we present new measurements for the scatter broadened quasar B 2005+403, combining Very Long Baseline Interferometry (VLBI) and flux density variability measurements obtained at various frequencies over the last decade. B $2005+403$ is a flat spectrum quasar $\left(S_{5 \mathrm{GHz}}=2.5-3.5 \mathrm{Jy}\right.$, $\alpha_{0.3 / 5 \mathrm{GHz}}=0.3$, with $S \sim v^{\alpha}$; Becker et al. 1991) at a redshift of $z=1.736$ (Boksenberg et al. 1976). It is located close to the Galactic plane at $l=76.82^{\circ}, b=4.29^{\circ}$ behind the Cygnus super-bubble region. Earlier studies showed that interstellar scattering affects the VLBI image of the source, causing angular broadening at frequencies below $5 \mathrm{GHz}$ (Fey et al. 1989; Mutel \& Lestrade 1990; Desai \& Fey 2001). The high value of the scattering measure $\left(\mathrm{SM}=\int_{0}^{L} C_{N}^{2}(s) \mathrm{d} s^{1}\right)$, derived for the line of sight of B $2005+403$ by Fey et al. (1989), reflects the strong influence of the interstellar medium.

In order to further investigate the scatter broadening and the amount of scintillation in B $2005+403$, we combined all available flux density monitoring data with our VLBI observations. The wide frequency coverage of our data facilitates a study of the interplay of the frequency dependent scattering effects, which dominate below $8 \mathrm{GHz}$, and the source intrinsic variability, which is best seen at and above $15 \mathrm{GHz}$. At these higher frequencies, B 2005+403 has not been intensively studied with VLBI. The VLBI data cover a time range of $11 \mathrm{yr}$ (from 1992-2003) and allow us to study and monitor the structural evolution of VLBI-core and pc-scale jet. The scatter broadening observed in the VLBI images at the lower frequencies is measured and gives further constraints to the properties of the intervening ISM. Additional parameters for the ISM are obtained from the IDV monitoring of the source, performed with the Effelsberg $100 \mathrm{~m}$ telescope.

The paper is organized as follows. In Sect. 2, we present details of the VLBI observations and corresponding data reduction and introduce the long-term trends of the flux density variability. In Sect. 3 we discuss the source structure, the kinematics of the inner jet components and the possible location of the nucleus (VLBI core). In Sect. 3.4, we focus on the results of the low frequency VLBI observations and discuss the scatter

\footnotetext{
1 The Scattering Measure is the path integral over the coefficient $C_{N}^{2}$ of the electron density fluctuation wavenumber spectrum.
}

broadening. In Sect. 3.5 we discuss the long-term variability of B $2005+403$ and relate it to changes in the VLBI structure. In Sect. 3.6 we discuss the implications the observed intraday variability. Section 4 gives a concluding summary.

The following cosmological parameters are used throughout this paper: $H_{0}=71 \mathrm{~km} \mathrm{~s}^{-1} \mathrm{Mpc}^{-1}, \Omega_{\mathrm{m}}=0.27$ and $\Omega_{\mathrm{vac}}=0.73$.

\section{Data and data reduction}

\subsection{VLBI data}

We analyzed 17 VLBI datasets of B 2005+403 from 14 different observing epochs obtained during 1992-2003. These observations were performed with different VLBI networks (EVN, VLBA, Global) at various frequencies ranging from $1.6 \mathrm{GHz}$ to $43 \mathrm{GHz}$. In our analysis we also included several data sets from the $2 \mathrm{~cm}$ survey (see Kellermann et al. 2004) and one observation of the source in the MOJAVE survey (Lister \& Homan 2005). In Table 1 we summarize the VLBI experiments.

After correlation at the VLBI correlators in Socorro (NRAO) or Bonn (MPIfR), the VLBI data were calibrated and fringe-fitted using the standard procedures within AIPS (Astronomical Image Processing System) software package. The post-processing of the data invoked the usual steps of editing, phase and amplitude self-calibration and imaging. These tasks were performed within AIPS and the Caltech DIFMAP packages. For previously published datasets (from the $2 \mathrm{~cm}$ survey and the MOJAVE survey and the datasets from epoch 2003.04), we reanalyzed the data. Starting from precalibrated visibilities, we repeated the self-calibration, imaging and Gaussian model fitting. For comparison of the results from this reanalysis with the original maps in the $2 \mathrm{~cm}$ Survey we refer the reader to Kellermann et al. (2004).

In three VLBI experiments the source was also observed in full polarization. Column 4 of Table 1 summarizes the recorded polarization. In the polarization analysis, the feed leakage terms for the antennas (D-terms) were determined in the usual way using the AIPS task "LPCAL" (Leppänen et al. 1995). At $15 \mathrm{GHz}$, we could use the University of Michigan (UMRAO) database to calibrate the orientation of the electric vector position angle (EVPA). We verified that total and correlated flux density (in total intensity and in polarization) matched within $10 \%$ accuracy, excluding a large miscalibration of the EVPA owing to undetected polarized large scale structure. At 22 and $43 \mathrm{GHz}$, we had no way to calibrate the EVPA. At these frequencies, the absolute orientation of the EVPA is therefore unknown.

In Fig. 1 we present the results from the VLBI imaging, showing the CLEAN maps obtained at $15 \mathrm{GHz}, 22 \mathrm{GHz}$, and $43 \mathrm{GHz}$. Figure 1 also shows the polarization images with the electric vectors superimposed.

To facilitate measurement of structural changes, the VLBI maps were parameterized in the usual manner using Gaussian components. We fitted Gaussian components to the calibrated visibility data using the DIFMAP program. The resulting modelfits are shown in a separate table which is available online. The table contains the following information: Col. 1 gives the 
Table 1. The epochs, frequencies and arrays of the VLBI observations described in this paper. In Col. 4 we indicated the polarization mode of the observation. In Col. 5 references for previously published data are given.

\begin{tabular}{|c|c|c|c|c|}
\hline Epoch & Frequency & Instrument & Pol. & Reference \\
\hline 1992.44 & $22 \mathrm{GHz}$ & EVN (8 stations) & LL & this paper \\
\hline 1994.17 & $22 \mathrm{GHz}$ & VLBA (8 stations) + VLA + EVN (4 stations) & LL & this paper \\
\hline 1995.27 & $15 \mathrm{GHz}$ & VLBA (2 cm Survey) & $\mathrm{RR}$ & Kellermann et al. (2004) \\
\hline 1995.96 & $15 \mathrm{GHz}$ & VLBA ( $2 \mathrm{~cm}$ Survey) & LL & Kellermann et al. (2004) \\
\hline 1996.73 & $43 \mathrm{GHz}$ & VLBA+EB & full & this paper \\
\hline 1996.73 & $22 \mathrm{GHz}$ & $\mathrm{VLBA}+\mathrm{EB}$ & full & this paper \\
\hline 1996.73 & $15 \mathrm{GHz}$ & VLBA+EB & full & this paper \\
\hline 1996.82 & $5 \mathrm{GHz}$ & $\mathrm{EVN}$ (8 stations) & LL & this paper \\
\hline 1996.83 & $8 \mathrm{GHz}$ & $\mathrm{EVN}$ (8 stations) & RR & this paper \\
\hline 1997.19 & $15 \mathrm{GHz}$ & VLBA ( $2 \mathrm{~cm}$ Survey) & LL & this paper \\
\hline 1998.14 & $1.6 \mathrm{GHz}$ & EVN (9 stations) & LL & this paper \\
\hline 1999.01 & $15 \mathrm{GHz}$ & VLBA ( $2 \mathrm{~cm}$ Survey) & LL & Kellermann et al. (2004) \\
\hline 2001.17 & $15 \mathrm{GHz}$ & VLBA ( $2 \mathrm{~cm}$ Survey) & LL & Kellermann et al. (2004) \\
\hline 2001.98 & $15 \mathrm{GHz}$ & VLBA ( $2 \mathrm{~cm}$ Survey) & LL & Kovalev et al. (2005) \\
\hline 2003.04 & $22 \mathrm{GHz}$ & VLBA & full & Bach (2004) \\
\hline 2003.04 & $15 \mathrm{GHz}$ & VLBA & full & Bach (2004) \\
\hline 2003.16 & $15 \mathrm{GHz}$ & VLBA (MOJAVE) & full & Lister \& Homan (2005); Kovalev et al. (2005) \\
\hline
\end{tabular}

observing epoch, frequency and VLBI array, Col. 2 identifies the VLBI components by letters and numbers, Col. 3 gives the flux density of each VLBI component $(S)$, Col. 4 its radial distance $r$ relative to component $\mathrm{C} 1$, Col. 5 the position angle $(\theta)$, Col. 6 the FWHM major axis $(a)$, Col. 7 the ratio of minor to major axis $(b / a)$, Col. 8 the position angle of the major axis $(\phi)$, and Col. 9 the reduced $\chi^{2}$ to characterize the goodness of the modelfit. The positional information (Cols. 4 and 5) are relative to the westernmost component, denoted as $\mathrm{C} 1$. It is further assumed that the position of $\mathrm{C} 1$ is stationary with time. The measurements' errors listed in the table were estimated from the internal scatter obtained during the process of imaging and model-fitting and is described in more detail e.g. in Krichbaum et al. (1998a). During the process of model fitting we reduced the number of independent variables by fitting only circular Gaussian components. For those cases where circular Gaussian model fits gave unsatisfactory results and produced unacceptably large reduced $\chi^{2}$ values, elliptical Gaussian components were fitted. Most of the $22 \mathrm{GHz}$ and $43 \mathrm{GHz}$ data were fitted best with elliptical Gaussians.

In Fig. 2 we show the VLBI images resulting from the Gaussian model fitting. The circles seen in this figure denote the positions and sizes of the individual model fit components. The contour lines result from the convolution of the modelfit components with the observing beam and the addition of the underlying (noise limited) residuals.

\subsection{Total flux density monitoring}

B 2005+403 is included in the flux density monitoring programs performed at the University of Michigan Radio Astronomy Observatory (UMRAO) in the USA and at the Metsähovi Radio Observatory in Finland. This allows us to study the long-term flux density variability. In Fig. 7 we show the lightcurves at $5 \mathrm{GHz}, 8 \mathrm{GHz}$ and $15 \mathrm{GHz}$ (Aller et al. 1999) and at $22 \mathrm{GHz}$ and $37 \mathrm{GHz}$ (Teräsranta et al. 1998;
Teräsranta et al. 2004). The covered time range is 1990-2004. A more detailed discussion of the variability and its possible relation to the structural changes seen in the VLBI maps is given in Sect. 3.5.

In the context of ongoing systematic studies of Intra-Day Variable radio sources, B 2005+403 was observed with the Effelsberg 100 m radio telescope of the Max-Planck-Institut für Radioastronomie. During two observing runs especially dedicated to search for IDV in sources located in the Cygnus region (the observed sources are those studied by Fey et al. 1989; Desai \& Fey 2001), we measured the flux density variability of B $2005+403$ at $6 \mathrm{~cm}(4.85 \mathrm{GHz})$ and $18 \mathrm{~cm}(1.67 \mathrm{GHz})$ with the Effelsberg $100 \mathrm{~m}$ radio telescope. The observations were performed on November 30 and December 6, 2003. The flux densities were measured adopting the method of repeated and subsequently averaged cross-scans. The measurement method and the data analysis is described in more detail e.g. in Kraus et al. (2003). The duty cycle of the measurement resulted in 1-2 flux density measurements per source and hour. For calibration purposes, several steep spectrum sources were included and observed with the same duty cycle as the target source B $2005+403$. From these additional sources, the source B $2021+614$ showed the least variability and therefore could be used as a secondary calibrator to monitor and correct for residual short time-scale fluctuations of the antenna gain. The absolute flux density scale was determined from repeated observations of the primary calibrators NGC 7027, 3C 48 and 3C 286 and the flux density scale of (Baars et al. 1977; Ott et al. 1994).

\section{Results}

\subsection{Kinematics}

In Fig. 1 we show the VLBI CLEAN maps obtained at $15 \mathrm{GHz}$, $22 \mathrm{GHz}$, and $43 \mathrm{GHz}$. The corresponding maps of the Gaussian modelfits are presented in Fig. 2. 

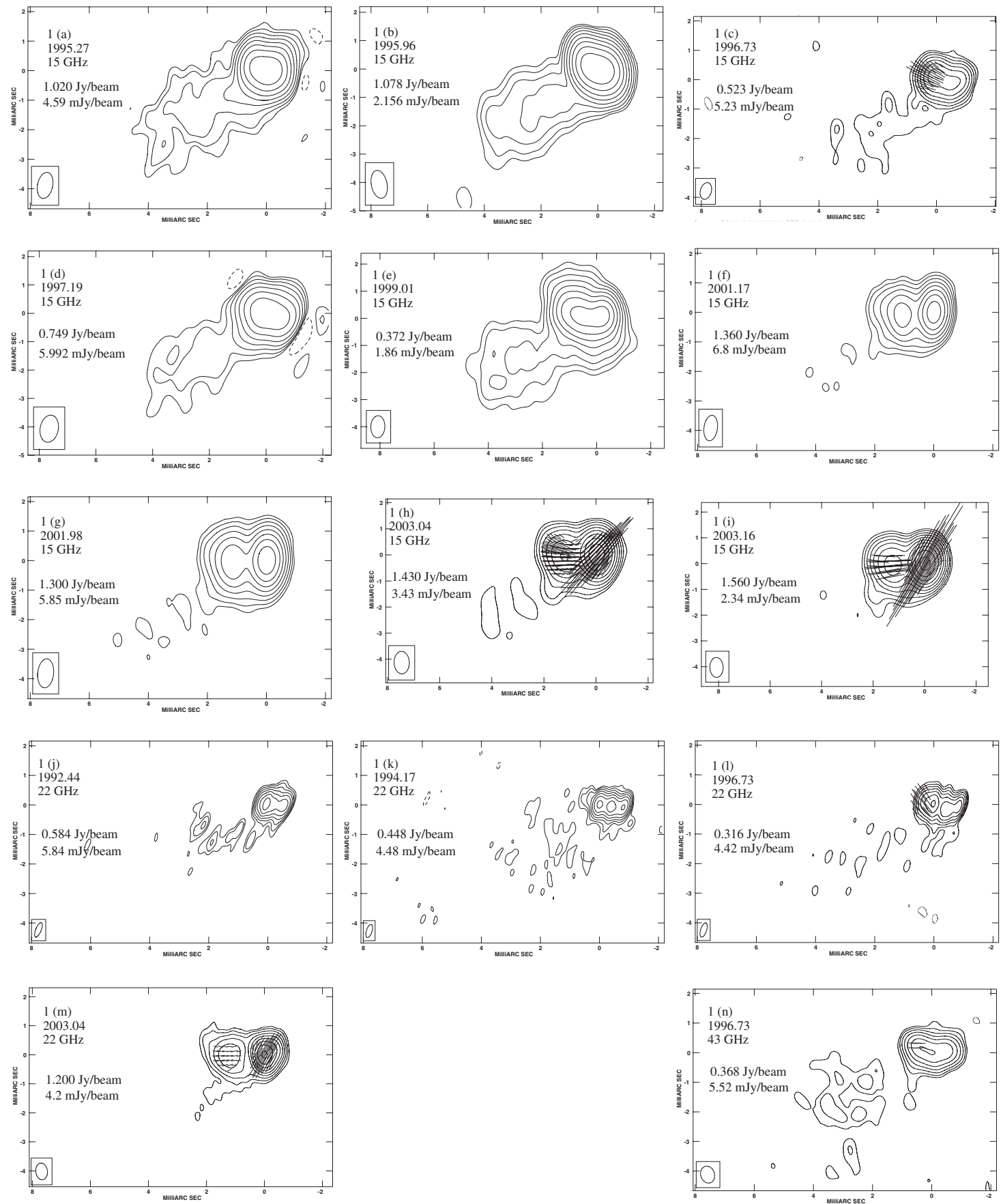

Fig. 1. VLBI CLEAN maps of B $2005+403$ at $15 \mathrm{GHz}, 22 \mathrm{GHz}$ and $43 \mathrm{GHz}$ shown at the different observing epochs. Epoch, frequency, peak flux density and the lowest (positive) contour are given in the upper left corner of each image. Contours are in percent of the peak flux and increase by factors of two. The beamsize is shown in the bottom left corner of each image. 1 mas length of the superimposed polarization vectors corresponds to $12.5,25,10,25,31.3,31.3 \mathrm{mJy} /$ beam in images (h) to (n), respectively. (Images were created in AIPS.)

The maps of B $2005+403$ show an east-west oriented corejet structure with several embedded components. The central 1 mas region is best described by three Gaussian components $\mathrm{C} 1, \mathrm{C} 2$, and $\mathrm{C} 3$. The relative alignment of these 3 components seems to vary over the time spanned by the observations. In most images, the relative alignment of $\mathrm{C} 1-\mathrm{C} 3$ suggests a slight bending to the north. Beyond 1 mas core separation and oriented more to the south-east, a faint and partially resolved region of diffuse and extended emission is visible.
It can be fitted by one or two Gaussian components of larger extent (C4 and $\mathrm{C} 5$ ).

In Fig. 3 we plot the relative separation from $\mathrm{C} 1$ for the components $\mathrm{C} 2$ to $\mathrm{C} 5$ versus time. Because of its inverted spectrum, compactness and variability, we assume that $\mathrm{C} 1$ is the VLBI core and that its position is suitable for use as a stationary reference point (Sect. 3.2 for a discussion). In the figure, we also superimpose a straight line resulting from a linear fit to the motion of each component. The slope of each line measures 

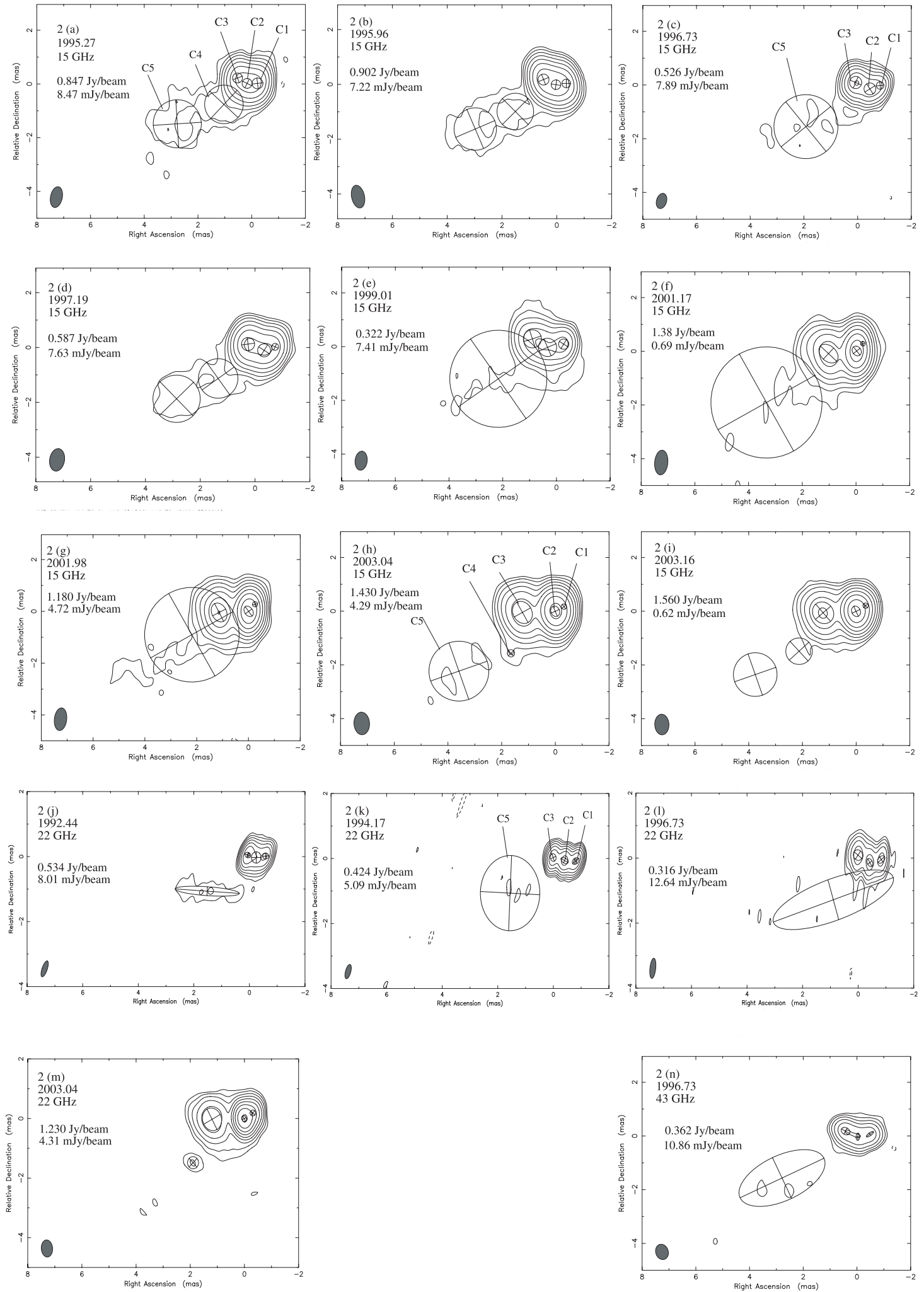

Fig. 2. Maps of Gaussian modelfits for B 2005+403. Circles characterize positions and sizes of the individual modelfit components. Contours result from the convolution with the observing beam. Epoch, frequency, peak flux density and the lowest (positive) contour level are given in the upper left corner of each image. Contours correspond to the modelfits, they are in percent of the peak flux and increase by factors of two. As in Fig. 1, the VLBI observations are at $15 \mathrm{GHz}, 22 \mathrm{GHz}$, and $43 \mathrm{GHz}$. (Images were created in DIFMAP.)

the angular separation rate. These rates and the corresponding apparent velocities are summarized in Table 3.
With the exception of component $\mathrm{C} 2$, which appears to be stationary within the measurement errors, the components $\mathrm{C} 3$ 

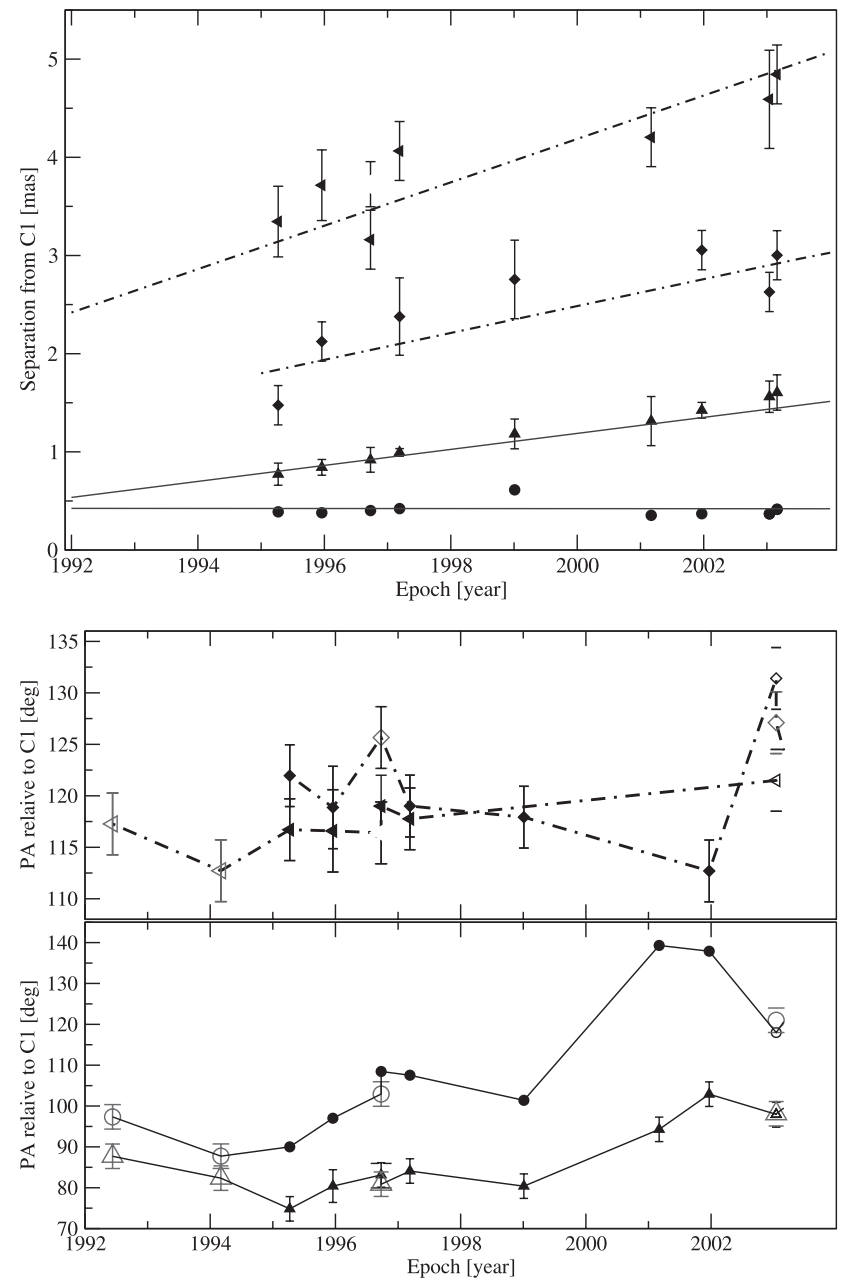

Fig. 3. Top panel: core separation as a function of time for components: C2 (circles), C3 (triangles), C4 (diamonds), C5 (left triangles). Filling of a symbol denotes the frequency of the observation: open for $22 \mathrm{GHz}$, filled for $15 \mathrm{GHz}$, larger and striped for $43 \mathrm{GHz}$. Solid lines indicate linear regression to reliable identifications ( $\mathrm{C} 2$ and $\mathrm{C} 3$ ). The dashed-dotted lines show regression to tentatively suggested identifications. Bottom panel: components position angle as a function of time. Same symbols and lines are used as above.

to C5 move with apparent superluminal velocities. For these three components we observe a systematic increase in their apparent velocities from $6.3 \mathrm{c}$ for component $\mathrm{C} 3$, to $9.9 \mathrm{c}$ for $\mathrm{C} 4$ and $16.8 \mathrm{c}$ for $\mathrm{C} 5$. In the $2 \mathrm{~cm}$ survey (Kellermann et al. 2004), B 2005+403 is characterized by a two component model, with the secondary component moving at an apparent speed of $12.3 \pm 3.0 \mathrm{c}$. Based on its core separation, this component may be identified with a blending of components $\mathrm{C} 4$ and C5 in our analysis.

The minimum Lorentz factor $\gamma_{\min }$ of the jet could be estimated from the maximum observed component speed. Adopting the usual equations for

$\gamma_{\min }=\sqrt{1+\beta_{\mathrm{app}}^{2}}$

and the inclination angle of the jet, which leads to maximum apparent speed

$\psi_{\gamma_{\min }}=\cot ^{-1} \beta_{\mathrm{app}}$
Table 3. The apparent speed and the corresponding apparent proper motion of components $\mathrm{C} 2, \mathrm{C} 3, \mathrm{C} 4$ and $\mathrm{C} 5$.

\begin{tabular}{ccc}
\hline \hline Component & $\begin{array}{c}\text { Apparent speed } \\
\operatorname{mas~yr}^{-1}\end{array}$ & $\begin{array}{c}\text { Apparent proper motion } \\
\text { in units of } c\end{array}$ \\
\hline C2 & $(-0.3 \pm 4.0) \times 10^{-3}$ & $\leq 0.31$ \\
C3 & $0.082 \pm 0.006$ & $6.25 \pm 0.46$ \\
C4 & $0.14 \pm 0.03$ & $9.91 \pm 2.12$ \\
C5 & $0.22 \pm 0.03$ & $16.77 \pm 2.29$ \\
\hline
\end{tabular}

one obtains for component C5 $\gamma_{\min } \geq 16.8 \pm 2.3$ and $\psi_{\gamma_{\min }}=$ $3.3^{\circ} \pm 0.5^{\circ}$.

Lähteenmäki \& Valtaoja (1999) calculated the Doppler boosting factor for a sample of active galactic nuclei using the total flux density monitoring data of the Metsähovi observatory. For B $2005+403$, they report a variability Doppler boosting factor of $\delta_{\mathrm{var}}=8.63$, which translates into $\delta_{\mathrm{var}}=10.9$, for the cosmological parameters used in this paper. We note that because of the light travel time argument used by Lähteenmäki \& Valtaoja (1999), the derived Doppler-factor is a lower limit of the true Doppler-factor. With the proper motions obtained from VLBI, we can calculate the viewing angle and the Lorentz factor of the jet:

$\psi_{\mathrm{var}}=\arctan \frac{2 \beta_{\mathrm{app}}}{\beta_{\mathrm{app}}^{2}+\delta_{\mathrm{var}}^{2}-1}$

$\gamma_{\mathrm{var}}=\frac{\beta_{\mathrm{app}}^{2}+\delta_{\mathrm{var}}^{2}+1}{2 \delta_{\mathrm{var}}}$

$\psi_{\mathrm{var}} \leq 4.8^{\circ} \pm 0.9^{\circ}$ and $\gamma_{\mathrm{var}} \geq 18.4 \pm 3.5$.

The apparent acceleration observed for components $\mathrm{C} 2$ to C5 may either result from an intrinsic acceleration of the jet, from a systematic bending of the jet, or from a combination of both effects. As $\psi_{\gamma_{\min }}$ and $\psi_{\text {var }}$ both are very small, a jet bending of less than a few degrees could easily explain the observed increase of the apparent velocities. To investigate this further, we plot in the lower panel of Fig. 3 the apparent variations of the position angle of the radius vector for each component. The plot indicates that component $\mathrm{C} 2$ and $\mathrm{C} 3$ move along similar and spatially bent trajectories with position angle changes in the range of $\sim 20-40^{\circ}$. Between 1992 and 2003 the position angle difference between $\mathrm{C} 2$ and $\mathrm{C} 3$ increases, indicating still similar but spatially offset paths. For components C4 and C5 the situation is less clear, as much smaller variations of the position angles are observed and the scatter in the data is larger. We therefore conclude that at least the components C2 and C3 move on non-ballistic and spatially bent trajectories.

The variation of the flux density of the individual jet components is plotted versus time in Fig. 4. Since most data are available at $15 \mathrm{GHz}$, we connect the flux density measurements at this frequency with lines. The variations of the flux density of components $\mathrm{C} 1, \mathrm{C} 2$ and $\mathrm{C} 3$ appear completely uncorrelated, with the flux density of $\mathrm{C} 1$ mainly decreasing, that of $\mathrm{C} 2$ increasing and the flux density of $\mathrm{C} 3$ showing some oscillatory behavior with at least two local maxima. In Sect. 3.5 we will discuss the combined flux density variability of the VLBI components with the variations seen in the total flux density of the source. 


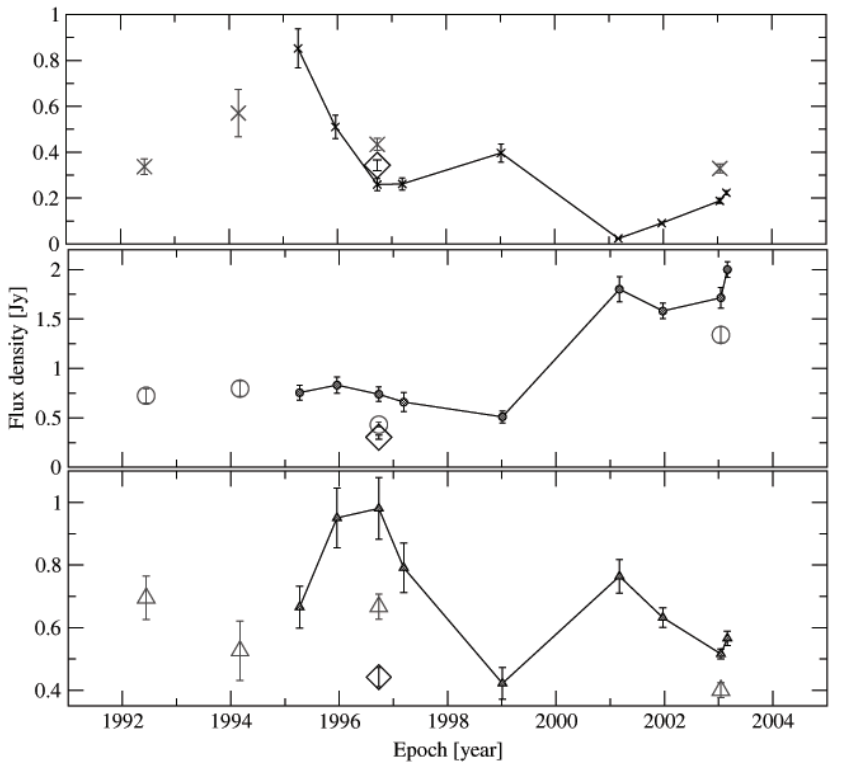

Fig. 4. Flux density evolution of individual jet components $\mathrm{C} 1, \mathrm{C} 2$ and $\mathrm{C} 3$ is shown. $\mathrm{C} 1$ is assumed to be the core. Open grey symbols denote observations at $22 \mathrm{GHz}$, filled black symbols at $15 \mathrm{GHz}$ and open diamonds represent the observation at $43 \mathrm{GHz}$.

\subsection{The position of the VLBI core}

From our simultaneous multi-frequency VLBI observations, the identification of the VLBI core and the measurement of individual component spectra is facilitated. For those components, where quasi-simultaneous flux density measurements were available at different frequencies, we calculated the spectral indices and show the results in Table 4. In 1996.73 and 2003.04, component $\mathrm{C} 1$ shows an inverted spectrum between $15 \mathrm{GHz}$ and $22 \mathrm{GHz}$. Between 22 and $43 \mathrm{GHz}$, the spectrum steepens. Components $\mathrm{C} 2$ and $\mathrm{C} 3$ both show an optically-thin synchrotron spectrum, with similar spectral indices of $\sim-0.7$ consistently measured in both observations. The spectral shape of $\mathrm{C} 1$ can be explained via synchrotronself absorption. This, its flux density variability and the high compactness seen in the modelfits indicate that $\mathrm{C} 1$ is the synchrotron-self absorbed jet base, which commonly is called the VLBI core.

Despite its optically thin spectrum, component $\mathrm{C} 2$ shows the most pronounced flux density variability (see Fig. 4). This is somewhat atypical, as pronounced flux density variability is usually seen in jet components with much flatter spectra. The apparent flux density increase of $\Delta S_{15 \mathrm{GHz}}=1.3 \mathrm{Jy}$ in $\Delta t_{\mathrm{var}}=2.16 \mathrm{yr}$ leads via the light travel time argument to an apparent brightness temperature of $T_{\mathrm{B}}=2.2 \times 10^{12} \mathrm{~K}$, which apparently violates the inverse Compton limit. The calculation leads to a lower limit for the Doppler factor of $\delta \geq 3.6$, which would be required to reduce the observed brightness temperature to $10^{12} \mathrm{~K}$. It is therefore very likely that the variability is caused mainly by differential Doppler boosting in combination with motion along a curved path. The relatively large variability and the apparent stationarity of $\mathrm{C} 2$ strongly indicate motion almost directly towards the observer, i.e. an angle to the line of sight close to zero. A similar interpretation to
Table 4. Spectral indices of the components C1, C2, and C3 $\left(S \sim v^{+\alpha}\right)$.

\begin{tabular}{c|ccc}
\hline \hline Epoch & C1 & C2 & C3 \\
\hline 1996.73 & $\alpha_{15}^{22}=1.34$ & $\alpha_{15}^{43}=-0.70$ & $\alpha_{15}^{43}=-0.70$ \\
& $\alpha_{22}^{43}=-0.35$ & & \\
\hline 2003.04 & $\alpha_{15}^{22}=1.46$ & $\alpha_{15}^{22}=-0.65$ & $\alpha_{15}^{22}=-0.67$ \\
\hline
\end{tabular}

explain apparent stationarity has been made for other sources, i.e. for 4C 39.25 (Alberdi et al. 2000).

On the basis of their spectral shapes with a steep spectral index of -0.7 , we rule out that components $\mathrm{C} 2$ or $\mathrm{C} 3$ can be identified with the VLBI core. An implication of this is that B 2005+403 is a one-sided core-jet source and that it exhibits no counter-jet. The lack of a counter-jet is usually explained as a consequence of Doppler boosting, consistent with our observations.

The spectral shape of $\mathrm{C} 1$ indicates the existence of a spectral maximum near $22 \mathrm{GHz}$ (Table 4). If this is identified with the synchrotron turnover of a homogeneous synchrotron self-absorbed component, we can estimate the magnetic field strength using the measured size of component $\mathrm{C} 1$ : 0.34 mas.

Following (e.g. Marscher 1983), the magnetic field strength is:

$B=10^{-9} \delta \frac{b(\alpha) \theta^{4} v^{5}}{S^{2}(1+z)} T$

where $\delta$ is the Doppler factor, $\theta$ is the component size in units of mas, $v$ is the turnover frequency in $\mathrm{GHz}, S$ (in Jy) is the flux density at the turnover frequency and $b(\alpha)$ is a spectral index dependent tabulated parameter (see Table 1 in Marscher 1983 ). Here we adopt $\alpha=-0.35$. This yields $B=1.7 \delta \times 10^{-4} T$ for $\mathrm{C} 1$.

\subsection{Polarization}

In Fig. 1 the polarization vectors are superimposed on the VLBI maps for those epochs where we have polarization data. The lengths of the vectors represent the polarized flux density. The polarized flux and the polarization angles derived from the VLBI observations are displayed in Table 5. Owing to the lack of absolute polarization angle calibration at $22 \mathrm{GHz}$ and $43 \mathrm{GHz}$, the position angles of the electric vectors are arbitrary at these frequencies. The measured polarization characteristics at $15 \mathrm{GHz}$ are in agreement with the UMRAO single dish measurements.

Component C2 was unpolarized in 1996.73, however this was the most prominent polarized feature in 2003.04 and 2003.16, with comparable polarized flux density at $15 \mathrm{GHz}$ and $22 \mathrm{GHz}$. The polarized flux density of C3 decreased through all the epochs and it also decreased with increasing frequency.

The polarization angles of $\mathrm{C} 2$ did not change significantly with time. The polarization angle of $\mathrm{C} 3$ changed by $\approx 15^{\circ}$ and $40^{\circ}$, at $15 \mathrm{GHz}$, and $22 \mathrm{GHz}$ respectively, from 1996.73 to 2003.04. In 2003.04 and 2003.16 it remained at the same value, roughly parallel to the jet direction implying that the 
Table 5. Polarization characteristics of B $2005+403$ obtained from the VLBI maps. Column 1 gives the epoch, Col. 2 the frequency of the observation. Column 3 gives the name of the component. Column 4 gives the polarized flux density in $\mathrm{mJy}$, Col. 5 the polarization angle in degrees. The EVPA is not calibrated the $22 \mathrm{GHz}$ and $43 \mathrm{GHz}$ datasets; the given values are arbitrary.

\begin{tabular}{ccccc}
\hline \hline Epoch & $v[\mathrm{GHz}]$ & ID & $P[\mathrm{mJy}]$ & $\chi\left(^{\circ}\right)$ \\
\hline 2003.04 & 15 & C2 & $66 \pm 14$ & $131 \pm 3$ \\
2003.04 & 22 & C2 & $74 \pm 14$ & $131 \pm 3$ \\
2003.16 & 15 & C2 & $67 \pm 14$ & $121 \pm 6$ \\
\hline 1996.73 & 15 & C3 & $42 \pm 5$ & $75 \pm 8$ \\
1996.73 & 22 & C3 & $35 \pm 4$ & $49 \pm 8$ \\
1996.73 & 43 & C3 & $13 \pm 3$ & $90 \pm 4$ \\
2003.04 & 15 & C3 & $38 \pm 4$ & $90 \pm 4$ \\
2003.04 & 22 & C3 & $21 \pm 3$ & $90 \pm 4$ \\
2003.16 & 15 & C3 & $28 \pm 5$ & $90 \pm 5$ \\
\hline
\end{tabular}

magnetic field is perpendicular to the jet, as one expects from an optically thin component.

The absolute angle of polarization is unknown at $22 \mathrm{GHz}$ and $43 \mathrm{GHz}$, however the angles in all epochs for both components are not significantly different from the calibrated EVPA at $15 \mathrm{GHz}$ (one exception is 1996.73 at $22 \mathrm{GHz}$ ). This is consistent with the low Rotation Measure $^{2}(\mathrm{RM})$ values determined by Zavala \& Taylor (2003).

\subsection{Low frequency VLBI observations}

The central part (denoted as M1 in Table 2) of B 2005+403 cannot be resolved at $1.6 \mathrm{GHz}, 5 \mathrm{GHz}$, and $8 \mathrm{GHz}$. However, at $5 \mathrm{GHz}$ and $8 \mathrm{GHz}$ two and one extended features appear in the jet direction, towards south-east (M2 and M3). M2 can be tentatively identified with $\mathrm{C} 5$ in the $15 \mathrm{GHz}$ and $22 \mathrm{GHz}$ data.

Our low frequency VLBI observations at $1.6 \mathrm{GHz}, 5 \mathrm{GHz}$ and $8 \mathrm{GHz}$ confirm the effect of angular broadening reported earlier by Mutel \& Lestrade (1990), Fey et al. (1989) and Desai \& Fey (2001). In Fig. 5 we show the $1.6 \mathrm{GHz}$ map obtained with the EVN in 1998.14. The image illustrates the effect of scatter broadening: the measured source size (36 mas) is three times larger than the size of the observing beam (13 mas). The possible elongation seen at this map at the position angle of $\sim 37^{\circ}$ may indicate anisotropy in the scattering.

Similar to Fey et al. (1989), we analyze the measured total angular source size as a function of the observing frequency. For this, we fitted the visibility data of B 2005+403 at all available frequencies with one Gaussian component. In Fig. 6 we plot the FWHM size of this component versus frequency, using our data obtained at $1.6 \mathrm{GHz}, 5 \mathrm{GHz}, 8 \mathrm{GHz}, 15 \mathrm{GHz}, 22 \mathrm{GHz}$ and $43 \mathrm{GHz}$ and including the previous measurements from the literature (Fey et al. 1989; Mutel \& Lestrade 1990; Desai \& Fey 2001); the corresponding values are tabulated in Table 6. The figure clearly shows a deviation from a simple power-law. In order to quantify the scatter broadening, which dominates

\footnotetext{
${ }^{2} \mathrm{RM}$ is an integration of magnetic field strength multiplied by the electron density along the line of sight from the source to the observer: $R M=812 \int_{0}^{L} n_{\mathrm{e}} B_{\|} \mathrm{d} l \mathrm{rad} \mathrm{m}^{-2}$, where $B_{\|}$is the parallel component of the magnetic field in $\mathrm{mG}$.
}

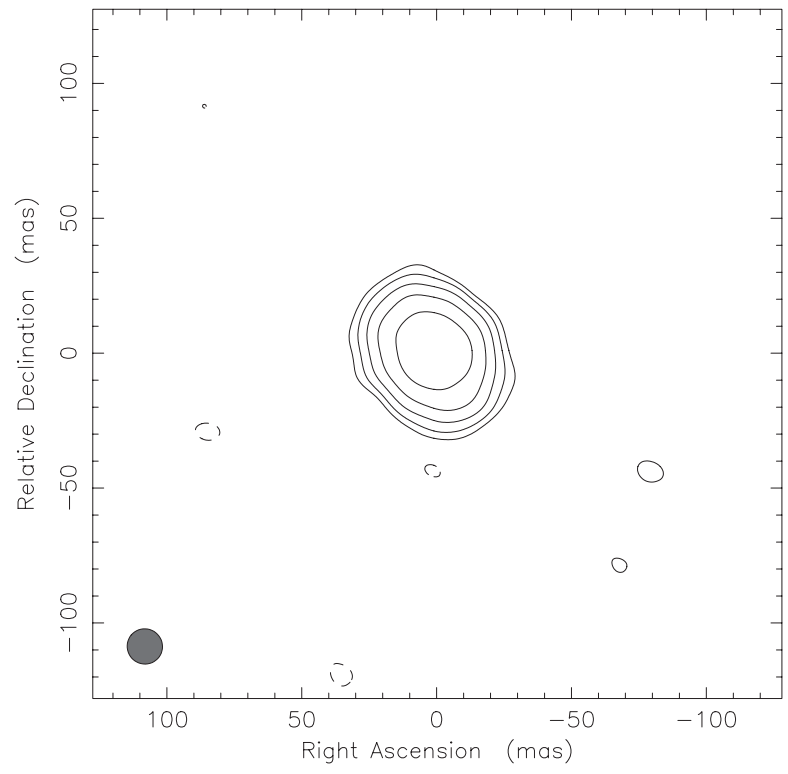

Fig. 5. Clean map obtained from the EVN observation (at $1.64 \mathrm{GHz}$ ) in 1998.14. The peak flux density is $0.464 \mathrm{Jy} / \mathrm{beam}$. The contour levels are $4.64 \mathrm{mJy} /$ beam $\times(-3.2,3.2,6.4,12.8,25.6,51.2)$. The beam FWHM is 13 mas, in contrast to the apparent angular size of the source of 36 mas.

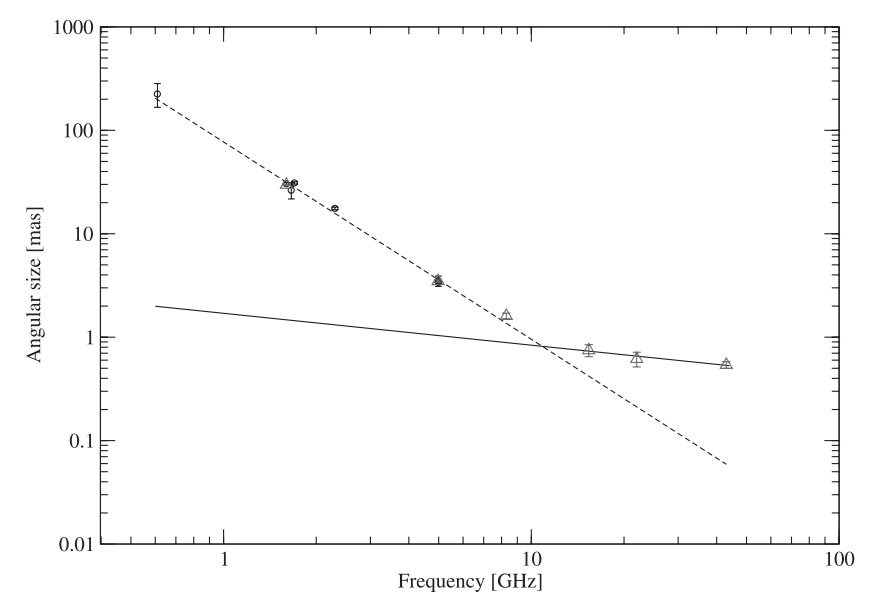

Fig. 6. The measured angular size plotted versus observing frequency. The dashed line represents a power law fit to the data in the range of $0.6 \mathrm{GHz}$ to $8 \mathrm{GHz}$. The slope of the line is $-1.91 \pm 0.05$. The solid line represents a power law with a slope of -0.31 , fitted to the data points at the high frequencies in the range of $15 \mathrm{GHz}$ to $43 \mathrm{GHz}$. Circles denote data from the literature, triangles data from this paper.

at lower frequencies, we fitted a power law to the data in the frequency range of $0.67-8 \mathrm{GHz}$. In this range we obtain the following result:

$\theta=(77.1 \pm 4.0) \cdot(v / 1 \mathrm{GHz})^{-(1.91 \pm 0.05)}$.

The dashed line in Fig. 6 shows this fit. We note that the slope does not change significantly if the data points at $8 \mathrm{GHz}$ are excluded from the fit. In this case one obtains: $\theta=(77.7 \pm 4.0)$. $(v / 1 \mathrm{GHz})^{-1.92 \pm 0.06}$. In both cases the slope of the power-law differs significantly from the slope of -2.2 one would expect for Kolmogorov type density fluctuations in the ISM. 
Table 6. The measured angular sizes of B $2005+403$ at different frequencies. Column 1 shows the observing frequency, Col. 2 the observing epoch, Col. 3 the major axis size of the fitted Gaussian component. In Col. 4 references for previously published data are given.

\begin{tabular}{cccc}
\hline \hline$v[\mathrm{GHz}]$ & Epoch & $\theta[\mathrm{mas}]$ & Reference \\
\hline 0.61 & Oct. 1986 & $225 \pm 58$ & Fey et al. (1989) \\
1.6 & 1998.14 & $29.8 \pm 0.5$ & this paper \\
1.66 & Mar. 1986 & $26.4 \pm 4.7$ & Fey et al. (1989) \\
1.7 & Jan. 1997 & $31 \pm 0.8$ & Desai \& Fey (2001) \\
2.3 & Jan. 1997 & $17.6 \pm 0.5$ & Desai \& Fey (2001) \\
4.99 & Oct. 1985 & $3.5 \pm 0.4$ & Fey et al. (1989) \\
5 & 1996.82 & $3.5 \pm 0.1$ & this paper \\
5 & Jan. 1997 & $3.4 \pm 0.1$ & Desai \& Fey $(2001)$ \\
8 & 1996.83 & $1.6 \pm 0.1$ & this paper \\
15 & 1996.73 & $0.8 \pm 0.1$ & this paper \\
22 & 1996.73 & $0.6 \pm 0.1$ & this paper \\
43 & 1996.73 & $0.5 \pm 0.04$ & this paper \\
\hline
\end{tabular}

Above $8 \mathrm{GHz}$ the source is significantly larger than the extrapolated scattering size. This indicates that towards higher frequencies scattering effects are less dominant and that the intrinsic structure of the sources is visible. The differences between the extrapolated scattering size and the measured source size are as follows: $\Delta \theta_{15 \mathrm{GHz}}=(0.4 \pm 0.1)$ mas, $\Delta \theta_{22 \mathrm{GHz}}=$ $(0.4 \pm 0.1)$ mas, and $\Delta \theta_{43 \mathrm{GHz}}=(0.4 \pm 0.1)$ mas. For the three size measurements above $8 \mathrm{GHz}$, we fit another power law which to first order can be used to characterize the frequency dependence of the intrinsic source size: $\theta_{\text {int }}=(1.7 \pm$ $0.4) \cdot(v / 1 \mathrm{GHz})^{-(0.31 \pm 0.07)}$ mas. The solid line in Fig. 6 shows this fit. With this curve we are able to obtain an upper limit of the intrinsic source size also at lower frequencies, where direct size measurements with VLBI are not possible.

Because of its heavily scattered line of sight, B 2005+403 was incorporated by Cordes and Lazio in their model describing the distribution of the Galactic free electrons (Cordes \& Lazio 2002). To account for the regions of intense scattering, they included "clumps", which are regions of enhanced electron density and/or electron density fluctuation. Adopting the scattering measure of Fey et al. (1989), a thickness $d \approx 18 \mathrm{pc}$ located at a distance of $L \approx 2.35 \mathrm{kpc}$ was derived. In their model, a Kolmogorov spectrum of interstellar turbulence was assumed.

At a given frequency, the size of the scattering disk can be estimated via the deconvolution formula: $\theta_{\text {scat }}=\sqrt{\theta_{\mathrm{obs}}^{2}-\theta_{\mathrm{int}}^{2}}$. For the scattering size at $1 \mathrm{GHz}$, we obtain $\theta_{1 \mathrm{GHz}}=(77.1 \pm$ 4.0) mas. Following Taylor \& Cordes (1993) and assuming Kolmogorov turbulence, we obtain for the scattering measure:

$\mathrm{SM}=\left(\frac{\theta_{\text {scat }}}{128 \mathrm{mas}}\right)^{5 / 3} \cdot v_{\mathrm{GHz}}^{11 / 3}$

The derived value of $\mathrm{SM}=(0.43 \pm 0.04) \mathrm{m}^{-20 / 3} \mathrm{kpc}$ is in good agreement and consistent with the previous measurement of Fey et al. (1989), however it now has a smaller uncertainty. Our slightly lower scattering measure implies either a somewhat reduced electron density fluctuation than that given in the Cordes \& Lazio (2002) model or a shorter path length through the screen. For the electron density fluctuation as given in
Cordes \& Lazio (2002), we formally obtain a path length of $11.7 \mathrm{pc}$, instead of the $18 \mathrm{pc}$ mentioned above.

Spangler \& Reynolds (1990) report the measurement of $\mathrm{H} \alpha$ intensity towards the Cygnus region. They studied lines of sight towards extragalactic radio sources which are affected by interstellar scattering. In the direction of B 2005+403, the reported $\mathrm{H} \alpha$ intensity is 130 Rayleigh, which corresponds to an emission measure of $E M=293.8 \mathrm{~cm}^{-6} \mathrm{pc}$ (assuming an electron temperature of $8000 \mathrm{~K}$ ). The EM corresponds to the path integral of the squared electron density along the line of sight. For a thin screen it can be approximated by: $E M=n_{\mathrm{e}}^{2} \cdot d$. Assuming that the probed HII region causes the scatter broadening using Eqs. (16) and (18) from Cordes \& Lazio (2002) we calculated from the EM and SM values the outer scale to be $l_{0}=25.5 \mathrm{AU}$, and the fractional variance of the electron density to be 6.9. Using the thickness of $11.7 \mathrm{pc}$ the electron density can be calculated: $n_{\mathrm{e}}=5.0 \mathrm{~cm}^{-3}$.

The exponent of the power-law of the angular broadening $(-1.91 \pm 0.05)$ indicates a wavenumber spectrum of the electron density turbulence, which is flatter than the usually assumed Kolmogorov type turbulence with a spectral slope of -2.2 . This can be produced in a medium consisting of random superpositions of discontinuities such as a collection of shock fronts (Rickett 1990). Romani et al. (1986) tabulate numerical estimates of scintillation parameters for different power-law spectra of electron density fluctuations. We used the equation with a power law index of -2 from Table 1 of Romani et al. (1986) that describes most adequately our angular broadening observation. From this we obtain $C_{N}^{2} d=15.16 \mathrm{~m}^{-20 / 3} \mathrm{kpc}$. With a thickness of the screen in the range of $d=12-18$ pc we obtain a range for $C_{N}^{2}=$ $(842 \ldots 1266) \mathrm{m}^{-20 / 3}$. However, we note that this is not an entirely consistent approach, as Cordes \& Lazio (2002) used a Kolmogorov spectrum.

From the power-law fitted to the source size at high frequencies, an upper limit for the intrinsic source size and hence lower limit for the brightness temperature can be calculated. At $1.6 \mathrm{GHz}, 5 \mathrm{GHz}$, and $8 \mathrm{GHz}$ the intrinsic source sizes are $\theta_{\text {int }} \leq(1.5 \pm 0.6)$ mas, $\theta_{\text {int }} \leq(1.0 \pm 0.3)$ mas, and $\theta_{\text {int }} \leq$ $(0.9 \pm 0.2)$ mas, respectively. The corresponding lower limits to the brightness temperatures are $T_{\mathrm{B}} \geq(0.6 \pm 0.5) \times 10^{12} \mathrm{~K}$, $T_{\mathrm{B}} \geq(0.14 \pm 0.09) \times 10^{12} \mathrm{~K}$ and $T_{\mathrm{B}} \geq(0.08 \pm 0.04) \times 10^{12} \mathrm{~K}$. These numbers are in accordance with typical brightness temperatures measured in VLBI (e.g. Kovalev et al. 2005) and neither strongly violate the inverse Compton limit nor indicate excessive Doppler-boosting.

\subsection{Long-term flux density variations}

In Fig. 7 we show the radio light-curves of B 2005+403 at $5 \mathrm{GHz}, 8 \mathrm{GHz}, 15 \mathrm{GHz}, 22 \mathrm{GHz}$ and $37 \mathrm{GHz}$ during the time interval covered by our VLBI observations. The data between $5 \mathrm{GHz}$ and $15 \mathrm{GHz}$ are from the Michigan (UMRAO) monitoring program, the data at $22 \mathrm{GHz}$ and $37 \mathrm{GHz}$ are from the Metsähovi monitoring.

The data clearly show the longterm variability of the source. A feature of particular interest is the apparent decrease 


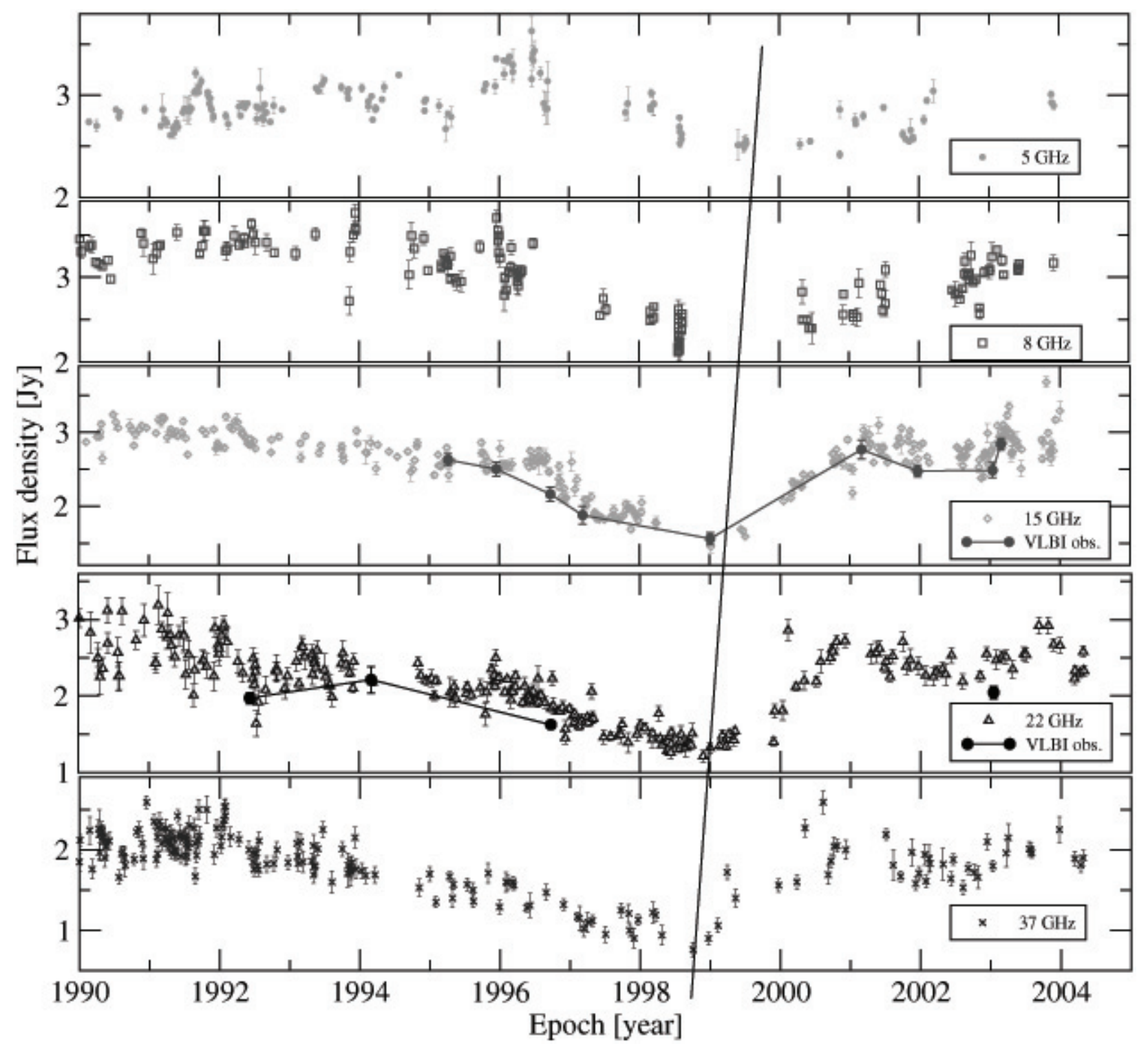

Fig. 7. Light-curves of B $2005+403$ at $5 \mathrm{GHz}, 8 \mathrm{GHz}, 15 \mathrm{GHz}, 22 \mathrm{GHz}$, and $37 \mathrm{GHz}$ from the monitoring data of the University of Michigan Radio Astronomy Observatory, and the Metsähovi $14 \mathrm{~m}$ radio telescope. The straight line connects the minima. The dark circles and lines denote the sum of flux density of the modelfit components from the VLBI observations at $15 \mathrm{GHz}$ and $22 \mathrm{GHz}$.

of the flux density observed between 1992 and 1999. Between 1999 and 2001, the flux increased again. This flux density "trough" is best seen and more pronounced at higher frequencies. At $5 \mathrm{GHz}$, the decline begins at 1996.5 and the flux density drops by $16 \%$, reaching its lowest value around 1999.4. At $8 \mathrm{GHz}$, a flux density decrease of $30 \%$ is observed during approximately the same time interval. At $15 \mathrm{GHz}$, the flux density drops by $47 \%$ from 1996.6 the beginning of 1999 . At the two highest observed frequencies $(22 \mathrm{GHz}$ and $37 \mathrm{GHz})$, the decrease is even more pronounced: $54 \%$ and $60 \%$ respectively. This systematic frequency dependence of the variability amplitude is accompanied by a systematic and frequency dependent shift of the time of the flux density minimum. The flux density minimum and the subsequent rise of the flux density appear earlier at higher frequencies. In Fig. 7 we illustrate this frequency dependence by a solid line, which is not a fit and only meant to guide the eye.
To quantify the variations at the different frequencies we use the modulation index, defined as follows:

$m=100 \frac{\sigma}{\langle S\rangle} \%$,

where $\sigma$ is the rms variation and $\langle S\rangle$ the mean flux density. The mean flux density and the corresponding variability indices are summarized in Table 7. At $22 \mathrm{GHz}$ the modulation index is largest.

In a number of AGN, short time flux density troughs are explained via an occultation by ISM clouds moving through the line of sight. These are the so-called "extreme scattering events" (ESE, see Fiedler et al. 1994). Depending on size, distance and relative velocity of the scatterer, timescales between a few days, months and perhaps even years are possible (Cimò et al. 2002; Pohl et al. 1995). Since B 2005+403 is located in a region of the sky with prominent scattering 
Table 7. The mean flux density, the standard deviation and the modulation indices for the flux density variability of B $2005+430$ at different frequencies between 1990 and 2004.

\begin{tabular}{cccc}
\hline \hline Frequency $(\mathrm{GHz})$ & $\langle S\rangle(\mathrm{Jy})$ & $\sigma$ & $m(\%)$ \\
\hline 5 & $2.86 \pm 0.02$ & 0.217 & 7.61 \\
8 & $3.03 \pm 0.02$ & 0.369 & 12.15 \\
15 & $2.63 \pm 0.02$ & 0.374 & 14.24 \\
22 & $1.95 \pm 0.03$ & 0.500 & 25.67 \\
37 & $1.84 \pm 0.03$ & 0.363 & 19.81 \\
\hline
\end{tabular}

effects, the interpretation of the observed flux density trough via such an extreme scattering event is a possible interpretation. However, the frequency dependence and variability time scales seen in B 2005+403 are different than those expected for an ESE. Typical ESE events show more pronounced and more rapid variations towards longer wavelengths, the opposite to what is observed in B 2005+403. Also the frequency dependence of the flux density minimum observed in the trough of B 2005+403 is not consistent with an ESE event, which owing to the geometrical occultation should not show any frequency shifts.

The observing dates of the low frequency VLBI observations performed in 1996.8 at $5 \mathrm{GHz}$ and 1998.1 at $1.6 \mathrm{GHz}$ coincide with the times of the decrease of the total flux density. The observed angular source sizes at these times, however, are not significantly different to the sizes observed previously $(1985,1986)$ by Fey et al. (1989) long before the flux density trough. This indicates that the amount of observed angular broadening is persistent and therefore is not related to the observed flux density variability.

In an alternative interpretation we argue that the observed flux density trough can be explained by the sum of the flux densities of the variable VLBI components. In Fig. 7 we display the sum of the flux density of the modelfit components $\mathrm{C} 1+\mathrm{C} 2+\mathrm{C} 3$ from the VLBI observations at $15 \mathrm{GHz}$ and $22 \mathrm{GHz}$. Particularly at $15 \mathrm{GHz}$, where the time coverage of the VLBI experiments covers the full time range of the trough, there is an excellent agreement between the single dish flux density measurements and summed VLBI component fluxes. Examining the flux densities of the individual components (see Fig. 4), it appears as if the combination of the declining trend of $\mathrm{C} 1$ and the increasing trend of $\mathrm{C} 2$ are mainly responsible for the shape of the flux density trough. We therefore conclude that the peculiar shape of the total flux density lightcurve of B $2005+403$ is a result of a blending of the independently varying flux of the inner jet components.

\subsection{Short time-scale flux density variations}

In November and December 2003 the flux density variability of B 2005+403 was monitored with the Effelsberg $100 \mathrm{~m}$ radio telescope with high time resolution. In Figs. 8 and 9 we plot the variability curves at $18 \mathrm{~cm}$ and $6 \mathrm{~cm}$, respectively.

In Table 8 we summarize the results from these measurements. To characterize the variability amplitudes and their significance we follow the methods described in Heeschen et al. (1987) and Kraus et al. (2003). Column 2 of the table gives

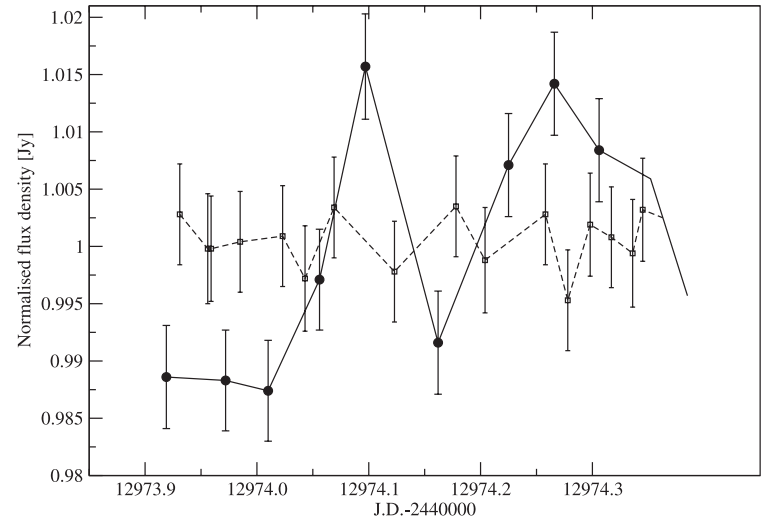

Fig. 8. Light-curve of B $2005+403$ at $1.6 \mathrm{GHz}$ in November 2003 observed with the Effelsberg $100 \mathrm{~m}$ telescope. Filled circles denote B 2005+403, open squares (connected with dashed line) the calibrators, NGC 7027 and B 2021+614. The modulation indices of the secondary calibrators are a measure of the calibration uncertainty, which is $m_{0} \leq 0.25 \%$. The modulation index of B $2005+403$ is $m=1.01 \%$.

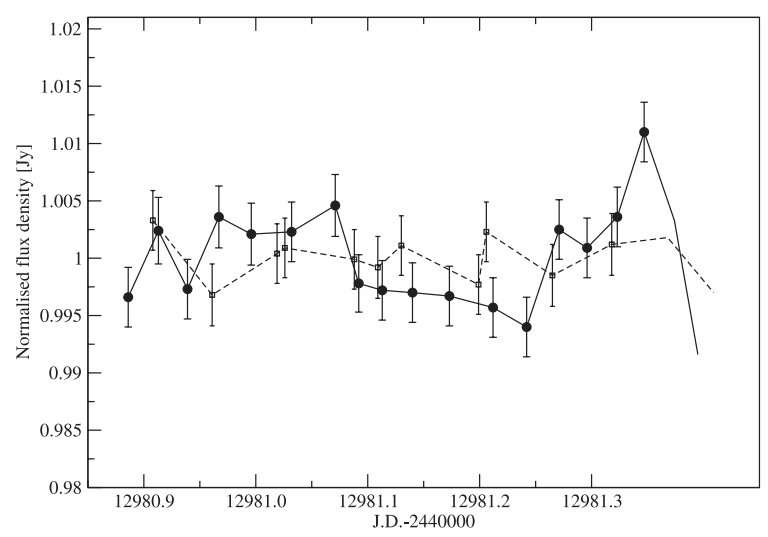

Fig. 9. Light-curve of B $2005+403$ at $5 \mathrm{GHz}$ in December 2003 observed with the Effelsberg $100 \mathrm{~m}$ telescope. Filled circles denote B $2005+403$, open squares (connected with dashed line) a calibrator, NGC 7027. The modulation index of the calibrator is $m_{0}=0.20 \%$, the modulation index of B $2005+403$ is $m=0.45 \%$.

the average flux density, Col. 3 the rms standard deviation, Col. 4 the modulation index, Col. 5 the variability amplitude (defined as $Y=3 \sqrt{m^{2}-m_{0}^{2}}$, see Kraus et al. 2003) and Col. 6 the reduced $\chi^{2}$. A value of $Y$ is only given, if the $\chi^{2}$-test gives a higher than 99.9 probability for significant variations. $m_{0}$ represents the maximum modulation index of the calibrators. From Table 8 and Figs. 8 and 9 the variability amplitude of B $2005+403$ decreases with frequency. Formally we measure a modulation index of $m=1.01 \%$ at $1.67 \mathrm{GHz}$ and of $m=0.45 \%$ at $5 \mathrm{GHz}$. At this frequency the detection of IDV is marginal. We note that the model of refractive interstellar scintillation in the weak regime predicts a decrease of the modulation index with increasing frequency (e.g. Rickett 1990). This is consistent with our observations.

The characteristic variability timescales derived from the light curves in Figs. 8 and 9 range between $t_{\mathrm{var}}=$ $0.09-0.13$ days at $18 \mathrm{~cm}$ and $t_{\mathrm{var}}=0.05-0.12$ days at $6 \mathrm{~cm}$. Adopting a source intrinsic interpretation for the variability we 
Table 8. The variability parameters of B $2005+403$ and the secondary calibrators at $1.67 \mathrm{GHz}$ (top) and $4.85 \mathrm{GHz}$ (bottom). Column 2 shows the mean flux density in Jy, Col. 3 its standard deviation, Col. 4 the modulation index, Col. 5 the noise-bias corrected variability amplitude and Col. 6 the reduced $\chi^{2}$.

\begin{tabular}{cccccc}
\hline \hline Source name & $\langle S\rangle(\mathrm{Jy})$ & $\sigma$ & $m(\%)$ & $Y(\%)$ & red. $\chi^{2}$ \\
\hline \multicolumn{7}{c}{$\lambda=18 \mathrm{~cm}, m_{0}=0.25 \%$} \\
\hline B 2005+403 2.430 & 0.024 & 1.01 & 2.93 & 5.593 \\
NGC 7027 & 1.906 & 0.004 & 0.19 & 0 & 0.197 \\
B 2021+614 & 2.193 & 0.006 & 0.25 & 0 & 0.365 \\
\hline \multicolumn{7}{c}{$\lambda=6 \mathrm{~cm}, m_{0}=0.20 \%$} \\
\hline B 2005+403 & 2.905 & 0.013 & 0.45 & 1.18 & 3.107 \\
NGC 7027 & 5.489 & 0.011 & 0.20 & 0 & 0.621 \\
\hline
\end{tabular}

may apply the light travel time argument and derive - via the source size - an apparent brightness temperature. Following Wagner et al. (1996) we obtain for the apparent "variability brightness temperature":

$T_{\mathrm{B}}=4.5 \times 10^{10} \Delta S\left(\frac{\lambda D_{\mathrm{L}}}{t_{\mathrm{var}}(1+z)^{2}}\right)^{2} \mathrm{~K}$

where $\Delta S$ is the amplitude of the flux density variations [Jy], $\lambda[\mathrm{cm}]$ the wavelength, $D_{\mathrm{L}}[\mathrm{Mpc}]$ the luminosity distance, and $t_{\mathrm{var}}$ [day] is the variability time-scale. The resulting apparent brightness temperatures is in the range of $T_{\mathrm{B}}=$ $10^{18 \ldots 21} \mathrm{~K}$. This can be reduced to the inverse-Compton limit with Doppler-factors in the range of a few hundred of to one thousand. Since these values appear unreasonably high and are not consistent with the results from VLBI nor with theoretical expectations (e.g. Begelman et al. 1994), we probably can rule out that source intrinsic effects are responsible for the observed rapid variations.

The source size and the apparent brightness temperature can be also determined from the interstellar scintillation model, assuming that the main reason for the observed variability is the motion of the Earth through the scintillation pattern (see for example Goodman 1997, and references therein). In this scenario the variability timescale (in [days]) is given by: $t_{\text {var }}=347.1 \theta_{\text {scat }} L v^{-1}$ with the scattering size $\theta_{\text {scat }}$ in [mas], the screen distance $L$ in $[\mathrm{kpc}]$ and the relative velocity between screen and earth $v$ in $\left[\mathrm{km} \mathrm{s}^{-1}\right]$. It is clear that a screen located at kpc distance as proposed by Cordes \& Lazio (2002) is not able to explain the observed short variability timescale. With a scattering size (at $1.67 \mathrm{GHz}$ ) of $\theta_{\text {scat }}=30$ mas, a screen distance of $2.4 \mathrm{kpc}$ and typical galactic velocities $v \leq 220 \mathrm{~km} \mathrm{~s}^{-1}$, one would expect to see variations on timescales $t_{\mathrm{var}}>100$ days.

In order to reproduce a variability time scale of 0.1 day at $1.67 \mathrm{GHz}$ we obtain with the above equation the following constraint for scattering size and screen distance: $\theta_{\text {scat }} L \leq$ $6.34 \times 10^{-2}$. For the measured scattering size of $\sim 30$ mas, this leads to unreasonable nearby screen distances of $\leq 2 \mathrm{pc}$. The only way to resolve this dilemma is a smaller scattering size. Adopting a minimum screen distance of at least 10-20 pc as required for the interpretation of the ultrafast scintillators (e.g. Dennett-Thorpe \& de Bruyn 2002, 2003), we would obtain a scattering size of $\theta_{\text {scat }} \leq 6.3$ mas. A source with such a size would have an apparent brightness temperature at $1.6 \mathrm{GHz}$ of
$T_{\mathrm{B}} \geq 10^{10} \mathrm{~K}$, fully consistent with our VLBI measurements (see Sect. 3.4).

An upper limit to the distance of the screen is obtained from the restriction of the source size via the inverse Compton limit of $T_{\mathrm{B}}^{\mathrm{IC}}=10^{12} \mathrm{~K}$. The requirement that the brightness temperature has to be lower than this leads to a source size of $\theta=\sqrt{1.22 \times 10^{12} S_{\nu} \nu^{-2} T_{\mathrm{B}}^{-1} \delta^{-1}(1+z)} \geq 0.5 \mathrm{mas}$, where we adopted $S_{1.6 \mathrm{GHz}}=2.4 \mathrm{Jy}$ from Table 8 and for the Doppler factor $\delta \simeq 10$. This leads with the above equations to an upper limit of the screen distance of $D \leq 0.58 \mathrm{v}$ pc or with $(10 \leq v \leq 220) \mathrm{km} \mathrm{s}^{-1}(6 \leq L \leq 127) \mathrm{pc}$.

We therefore conclude that the observed IDV can be explained by a screen, located at a distance of $L \leq 130$ pc that can be characterized by a scattering size of $0.5 \leq \theta_{\text {scat }} \leq 6.3$ mas. The corresponding scattering measure at $1.6 \mathrm{GHz}$ then is of the order of $6.4 \times 10^{-4} \leq S M \leq 4.3 \times 10^{-2}$, measured in the conventional units of SM. These values are considerably lower than the scattering measure of the more distant screen, which is responsible for the scatter broadening in B $2005+403$. We therefore conclude that the observed IDV is not caused by this distant screen. There is likely multiple scattering by at least two spatially and physically very different plasma screens. In this scenario the first screen leads to a significant scatter broadening of the source image, which in the second screen is only very weakly scattered due to large quenching effects $\left(\theta_{\text {source }} \gg \theta_{\text {scat }}\right)$. This quenched scattering (e.g. Rickett 1986) by the second screen could explain the relatively low variability amplitudes of $\leq 1 \%$ observed in our IDV experiments at 6 and $18 \mathrm{~cm}$. Quantitatively this can be verified using Eq. (20) of Goodman (1997), which relates the variablity index, the scattering measure, the effective source size and the screen distance. With the parameters above a modulation index of $m \leq 1 \%$ is obtained, in good agreement with our observations.

\section{Discussion and summary}

B 2005+403 is located in the Cygnus-region and previously was known as one of the most scattered broadened extragalactic sources in this region of the sky. In this paper we combined multi-frequency flux density monitoring and VLBI imaging observations. We also used high time resolution flux density monitoring observations to search for possible IntraDay Variability. The combination of these complementary data yields new and more accurate estimates of (i) the source structure and its kinematics and of (ii) the properties of the interstellar medium responsible for the observed propagation effects.

High frequency VLBI imaging observations (at 15-43 GHz) spanning 11 years (1992-2003) reveal a one sided and bent core jet structure, with at least 5 embedded VLBI components. The inner jet components separate from the stationary assumed core $\mathrm{C} 1$ with apparent superluminal speeds of 6-17 c. The component nearest to the core (C2), however, appeared stationary $\left(\beta_{\text {app }} \leq 0.3\right)$. The flux density variation of this stationary and steep spectrum component apparently violates the inverse Compton limit, indicating strong Doppler-boosting. This could be explained by a component path oriented at a smaller angle to the line of sight than those 
of the other jet components. A systematic increase of the component speed with increasing core separation is observed. The paths of the inner-most jet components are curved and their motion is not ballistic, suggesting a spatially curved (helical) jet. Beyond a 2 mas core-separation, however, the components seem to move on linear (ballistic) trajectories. Future VLBI monitoring will be necessary to determine their paths more accurately.

The VLBI images at longer wavelengths $(1.6-8 \mathrm{GHz})$ show a scatter-broadened, nearly point-like source. Combining our new size measurements with published data from earlier observations allowed us to study the frequency dependence of the source size in greater detail. Below $8 \mathrm{GHz}$ this dependence is best described by a non-Kolmogorov power law with slope of -1.9 . For the size of the scattering disk at $1 \mathrm{GHz}$ we obtain $(77.1 \pm 4.0)$ mas. Above $8 \mathrm{GHz}$ the measured sizes are increasingly larger than the prediction from the scattering law, and the internal source structure becomes visible. Based on the observed scatter broadening, several parameters of the scattering medium (scattering size, scattering measure, electron density) were determined and found to be in accordance with previously published estimates (NE2001 model, Cordes \& Lazio 2002). The NE2001 model places the scattering screen at a distance of $2.35 \mathrm{kpc}$. We however note that towards the direction of B $2005+403$ there is a degree-size extended supernova remnant G78.2+2.1. It has a patchy, inhomogeneous structure at optical, X-ray and radio wavelengths (Bykov et al. 2004, and references therein). Its distance is $(1.5 \pm 0.5) \mathrm{kpc}$ (Landecker et al. 1980), still compatible with the scattering model.

We discuss the observed long term flux density variability curves (5-37 GHz), which during 1996-2001 showed a remarkable flux density "trough". A tentative interpretation via a possible "scattering event", similar to the "extreme scattering events" observed in other sources, could be abandoned on the basis of the observed frequency dependence of the variability, which appears more pronounced at higher frequencies. Instead, we show that the "trough" is well explained by the summed flux density variability of the (independently) varying VLBI components (C1-C3). In contrast to other sources, which often show correlations between jet component ejection and flux density variability (e.g. Savolainen et al. 2002, and references therein), such a relation is not seen in B $2005+403$. In this source at least some (if not most) of the observed flux density variability results from the blending of the evolving jet components.

Densely time-sampled variability measurements performed with the $100 \mathrm{~m}$ Effelsberg telescope showed only weak intraday variability in B $2005+403$. This was in contrast to naive expectations in which more pronounced variability was anticipated. The low variability indices $(m=1 \%$ at $18 \mathrm{~cm}, m=0.5 \%$ at $6 \mathrm{~cm}$ ) and the short variability time scale of $\sim 0.1$ days cannot be explained by scattering effects from the kpc-screen, which is responsible for the scatter broadening. Instead, another and much closer scatterer is required. Using the thin screen approximation for refractive interstellar scintillation we determined the likely distance of the scattering screen to be $(6 \leq L \leq 128)$ pc. At $18 \mathrm{~cm}$, its scattering size would lie in the range of $0.5 \leq \theta_{\text {scat }} \leq 6.3$ mas. For B 2005+403 a scattered-broadened image (kpc-screen) is scattered a second time (pc-screen) and the combination of both effects limits the accuracy of low frequency flux density variability measurements to about $0.5-1 \%$ and the angular the resolution of VLBI observations to a few milliarcseconds. We note that weak refractive interstellar scintillation from a very nearby and ionized ISM (which most likely covers larger portions of the sky), may therefore also limit the calibration accuracy and sensitivity of planned large radio telescopes operating at low frequencies, like LOFAR or the square-kilometer array (SKA).

Acknowledgements. We thank M. Lister, and Hugh Aller and Margo Aller for kindly providing data prior to publication; Alan Roy, Thomas Beckert and Jacques Roland for scientific discussion and useful comments. This work is based on observations with the $100 \mathrm{~m}$ telescope of the MPIfR (Max-Planck-Institut für Radioastronomie) at Effelsberg. This research has made use of data from the University of Michigan Radio Astronomy Observatory which has been supported by the University of Michigan and the National Science Foundation. The European VLBI Network is a joint facility of European, Chinese, South African and other radio astronomy institutes funded by their national research councils. The National Radio Astronomy Observatory is a facility of the National Science Foundation operated under cooperative agreement by Associated Universities, Inc. U.B. was partly supported by the European Community's Human Potential Programme under contract HPRCN-CT-2002-00321. K.É.G. has been supported for this research through a stipend from the International Max Planck Research School (IMPRS) for Radio and Infrared Astronomy at the Universities of Bonn and Cologne.

\section{References}

Alberdi, A., Gómez, J. L., Marcaide, J. M., Marscher, A. P., \& Pérez-Torres, M. A. 2000, A\&A, 361, 529

Aller, M. F., Aller, H. D., Hughes, P. A., \& Latimer, G. E. 1999, ApJ, 512,601

Baars, J. W. M., Genzel, R., Pauliny-Toth, I. I. K., \& Witzel, A. 1977, A\&A, 61, 99

Bach, U. 2004, Ph.D. Thesis

Becker, R. H., White, R. L., \& Edwards, A. L. 1991, ApJS, 75, 1

Begelman, M. C., Rees, M. J., \& Sikora, M. 1994, ApJ, 429, L57

Boksenberg, A., Briggs, S. A., Carswell, R. F., Schmidt, M., \& Walsh, D. 1976, MNRAS, 177, 43P

Bykov, A. M., Krassilchtchikov, A. M., Uvarov, Y. A., et al. 2004, A\&A, 427, L21

Cimò, G., Beckert, T., Krichbaum, T. P., et al. 2002, Pub. Astron. Soc. Australia, 19, 10

Cordes, J. M., \& Lazio, T. J. W. 2002, preprint

[arXiv:astro-ph/0207156]

Dennet-Thorpe, J., \& de Bruyn, A. G. 2000, ApJ, 529, 65

Dennett-Thorpe, J., \& de Bruyn, A. G. 2002, Nature, 415, 57

Dennett-Thorpe, J., \& de Bruyn, A. G. 2003, A\&A, 404, 113

Desai, K. M., \& Fey, A. L. 2001, ApJS, 133, 395

Doeleman, S. S., Shen, Z.-Q., Rogers, A. E. E., et al. 2001, AJ, 121, 2610

Fey, A. L., Spangler, S. R., \& Mutel, R. L. 1989, ApJ, 337, 730

Fiedler, R., Dennison, B., Johnson, K. J., Waltman, E. B., \& Simon, R. 1994, ApJ, 430, 581

Goodman, J. 1997, New Astron., 2, 449

Heeschen, D. S., Krichbaum, T. P., Schalinski, C. J., \& Witzel, A. 1987, AJ, 94, 1493 
Kedziora-Chudczer, L. L., Jauncey, D. L., Wieringa, M. H., Tzioumis, A. K., \& Reynolds, J. E. 2001, MNRAS, 325, 1411

Kellermann, K. I., \& Pauliny-Toth, I. I. K. 1969, ApJ, 155, L71

Kellermann, K. I., Lister, M. L., Homan, D. C., et al. 2004, ApJ, 609, 539

Kovalev, Y. Y., Kellermann, K. I., Lister, D. C., et al. 2005, AJ, 130, 2473

Kraus, A., Krichbaum, T. P., Wegner, R., et al. 2003, A\&A, 401, 161

Krichbaum, T. P., Alef, W., Witzel, A., et al. 1998a, A\&A, 329, 873

Krichbaum, T. P., Graham, D. A., Witzel, A., et al. 1998b, A\&A, 335, L106

Krichbaum, T. P., Kraus, A., Fuhrmann, L., Cimò, G., \& Witzel, A. 2002, PASA, 19, 14

Lähteenmäki, A., \& Valtaoja, E. 1999, ApJ, 521, 493

Landecker, T. L., Roger, R. S., \& Higgs, L. A. 1980, A\&AS, 39, 133

Leppänen, K. J., Zensus, J. A., \& Diamond, P. J. 1995, AJ, 110, 2479

Lister, M. L., \& Homan, D. C. 2005, AJ, 130, 1389

Lo, K. Y., Shen, Z.-Q., Zhao, J.-H., \& Ho, P. T. P. 1998, ApJ, 508, L61

Lovell, J. E. J., Jauncey, D. L., Bignall, H. E., et al. 2003, AJ, 126, 1699

Macquart, J.-P., \& de Bruyn, G. 2005, ArXiv Astrophysics e-prints
Marscher, A. P. 1983, ApJ, 264, 296

Mutel, R. L., \& Lestrade, J.-F. 1990, ApJ, 349, 47

Ott, M., Witzel, A., Quirrenbach, A., et al. 1994, A\&A, 284, 331

Pohl, M., Reich, W., Krichbaum, T. P., et al. 1995, A\&A, 303, 383

Qian, S.-J., Kraus, A., Zhang, X.-Z., et al. 2002, ChJAA, 2, 325

Quirrenbach, A., Witzel, A., Wagner, S., et al. 1991, ApJ, 372, L71

Rickett, B. J. 1986, ApJ, 307, 564

Rickett, B. J. 1990, ARA\&A, 28, 561

Romani, R. W., Narayan, R., \& Blandford, R. 1986, MNRAS, 220, 19

Savolainen, T., Wiik, K., Valtaoja, E., Jorstad, S. G., \& Marscher, A. P. 2002, A\&A, 394, 851

Spangler, S. R., \& Reynolds, R. J. 1990, ApJ, 361, 116

Taylor, J. H., \& Cordes, J. M. 1993, ApJ, 411, 674

Teräsranta, H., Tornikoski, M., Mujunen, A., et al. 1998, A\&AS, 132, 305

Teräsranta, H., Achren, J., Hanski, M., et al. 2004, A\&A, 427, 769

Wagner, S. J., \& Witzel, A. 1995, ARA\&A, 33, 163

Wagner, S. J., Witzel, A., Heidt, J., et al. 1996, AJ, 111, 2187

Witzel, A., Heeschen, D. S., Schalinski, C., \& Krichbaum, T. 1986,

Mitteilungen der Astronomischen Gesellschaft Hamburg, 65, 239

Zavala, R. T., \& Taylor, G. B. 2003, ApJ, 589, 126 


\section{Online Material}


K. É. Gabányi et al.: High frequency VLBI observations of the scatter-broadened quasar B 2005+403, Online Material p 2

Table 2. The parameters of the Gaussian modelfits. Column 1 gives the observing epoch, the VLBI array and the frequency, Col. 2 the component identification. Column 3 shows the flux density of each component. In Col. 4, the relative separation to C1 and in Col. 5 the position angle are listed. Columns 6-8 list the major axis, the axial ratio of minor to major axis, and the major axis position angle. The last column shows the reduced $\chi^{2}$ of the model.

\begin{tabular}{|c|c|c|c|c|c|c|c|c|}
\hline $\begin{array}{l}\text { Epoch, array and frequency } \\
\text { (1) }\end{array}$ & $\begin{array}{l}\text { Comp. } \\
\text { (2) }\end{array}$ & $\begin{array}{l}S(\mathrm{Jy}) \\
(3)\end{array}$ & $\begin{array}{c}r \text { (mas) } \\
(4)\end{array}$ & $\begin{array}{l}\theta\left(^{\circ}\right) \\
(5)\end{array}$ & $\begin{array}{l}a \text { (mas) } \\
(6)\end{array}$ & $\begin{array}{l}b / a \\
(7)\end{array}$ & $\begin{array}{c}\phi\left(^{\circ}\right) \\
(8)\end{array}$ & $\begin{array}{l}\chi_{r}^{2} \\
(9)\end{array}$ \\
\hline $\begin{array}{c}1998.14 \\
\text { EVN } \\
1.6 \mathrm{GHz} \\
\text { Errors }\end{array}$ & M1 & $4 \%$ & 0.00 & - & $7 \%$ & 0.8 & $3^{\circ}$ & 4.5 \\
\hline $\begin{array}{c}1996.82 \\
\mathrm{EVN} \\
5 \mathrm{GHz} \\
\text { Errors }\end{array}$ & $\begin{array}{l}\text { M1 } \\
\text { M2 } \\
\text { M3 }\end{array}$ & $\begin{array}{c}2.572 \\
0.488 \\
0.069 \\
10 \%\end{array}$ & $\begin{array}{l}0.00 \\
2.67 \\
8.10 \\
12 \%\end{array}$ & $\begin{array}{c}- \\
129.7 \\
138.3 \\
4^{\circ}\end{array}$ & $\begin{array}{l}3.48 \\
3.24 \\
6.95 \\
5 \%\end{array}$ & $\begin{array}{l}0.70 \\
1.00 \\
1.00 \\
10 \%\end{array}$ & $\begin{array}{c}32.1 \\
- \\
- \\
4^{\circ}\end{array}$ & 2.6 \\
\hline $\begin{array}{l}1996.83 \\
\text { EVN } \\
8 \mathrm{GHz} \\
\text { Errors }\end{array}$ & $\begin{array}{l}\text { M1 } \\
\text { M2 }\end{array}$ & $\begin{array}{l}3.418 \\
0.297 \\
11 \%\end{array}$ & $\begin{array}{l}0.00 \\
3.06 \\
6 \%\end{array}$ & $\begin{array}{c}- \\
127.0 \\
3^{\circ}\end{array}$ & $\begin{array}{l}1.51 \\
2.13 \\
6 \%\end{array}$ & $\begin{array}{l}0.86 \\
0.69 \\
10 \%\end{array}$ & $\begin{array}{c}65.43 \\
44.7 \\
5^{\circ}\end{array}$ & 1.0 \\
\hline $\begin{array}{l}1995.27 \\
\text { VLBA } \\
15 \mathrm{GHz}\end{array}$ & $\begin{array}{l}\mathrm{C} 1 \\
\mathrm{C} 2 \\
\mathrm{C} 3 \\
\mathrm{C} 4 \\
\mathrm{C} 5\end{array}$ & $\begin{array}{c}0.852 \\
0.755 \\
0.666 \\
0.170 \\
0.185 \\
10 \%\end{array}$ & $\begin{array}{l}0.00 \\
0.39 \\
0.77 \\
1.78 \\
3.35 \\
14 \%\end{array}$ & $\begin{array}{c}- \\
90.0 \\
74.8 \\
122.0 \\
116.7 \\
3^{\circ}\end{array}$ & $\begin{array}{l}0.40 \\
0.37 \\
0.37 \\
1.44 \\
1.79 \\
12 \%\end{array}$ & $\begin{array}{l}1.00 \\
1.00 \\
1.00 \\
1.00 \\
1.00\end{array}$ & $\begin{array}{l}- \\
- \\
- \\
- \\
-\end{array}$ & 1.0 \\
\hline $\begin{array}{l}1995.96 \\
\text { VLBA } \\
15 \mathrm{GHz}\end{array}$ & $\begin{array}{l}\mathrm{C} 1 \\
\mathrm{C} 2 \\
\mathrm{C} 3 \\
\mathrm{C} 4 \\
\mathrm{C} 5\end{array}$ & $\begin{array}{c}0.510 \\
0.831 \\
0.951 \\
0.106 \\
0.104 \\
10 \%\end{array}$ & $\begin{array}{l}0.00 \\
0.38 \\
0.84 \\
2.12 \\
3.72 \\
12 \% \\
\end{array}$ & $\begin{array}{c}- \\
97.0 \\
80.4 \\
118.9 \\
116.6 \\
4^{\circ}\end{array}$ & $\begin{array}{l}0.31 \\
0.35 \\
0.40 \\
1.34 \\
1.55 \\
10 \% \\
\end{array}$ & $\begin{array}{l}1.00 \\
1.00 \\
1.00 \\
1.00 \\
1.00\end{array}$ & $\begin{array}{l}- \\
- \\
- \\
- \\
-\end{array}$ & 0.3 \\
\hline $\begin{array}{c}1996.73 \\
\text { VLBA+EB } \\
15 \mathrm{GHz} \\
\\
\text { Errors }\end{array}$ & $\begin{array}{l}\mathrm{C} 1 \\
\mathrm{C} 2 \\
\mathrm{C} 3 \\
\mathrm{C} 5\end{array}$ & $\begin{array}{c}0.260 \\
0.738 \\
0.981 \\
0.185 \\
10 \%\end{array}$ & $\begin{array}{l}0.00 \\
0.40 \\
0.92 \\
3.16 \\
18 \%\end{array}$ & $\begin{array}{c}- \\
108.5 \\
83.1 \\
119.0 \\
3^{\circ}\end{array}$ & $\begin{array}{l}0.28 \\
0.44 \\
0.45 \\
2.38 \\
10 \%\end{array}$ & $\begin{array}{l}1.00 \\
1.00 \\
1.00 \\
1.00\end{array}$ & $\begin{array}{l}- \\
- \\
- \\
-\end{array}$ & 0.3 \\
\hline $\begin{array}{c}1997.19 \\
\text { VLBA } \\
15 \mathrm{GHz}\end{array}$ & $\begin{array}{l}\mathrm{C} 1 \\
\mathrm{C} 2 \\
\mathrm{C} 3 \\
\mathrm{C} 4 \\
\mathrm{C} 5\end{array}$ & $\begin{array}{c}0.262 \\
0.659 \\
0.791 \\
0.075 \\
0.092 \\
15 \%\end{array}$ & $\begin{array}{l}0.00 \\
0.42 \\
0.99 \\
2.38 \\
4.07 \\
11 \%\end{array}$ & $\begin{array}{c}- \\
107.5 \\
84.1 \\
119.0 \\
117.8 \\
3^{\circ}\end{array}$ & $\begin{array}{c}0.25 \\
0.47 \\
0.48 \\
1.44 \\
1.73 \\
7 \%\end{array}$ & $\begin{array}{l}1.00 \\
1.00 \\
1.00 \\
1.00 \\
1.00\end{array}$ & $\begin{array}{l}- \\
- \\
- \\
- \\
-\end{array}$ & 2.0 \\
\hline $\begin{array}{c}1999.01 \\
\text { VLBA } \\
15 \mathrm{GHz} \\
\\
\text { Errors }\end{array}$ & $\begin{array}{l}\mathrm{C} 1 \\
\mathrm{C} 2 \\
\mathrm{C} 3 \\
\mathrm{C} 4\end{array}$ & $\begin{array}{c}0.396 \\
0.510 \\
0.422 \\
0.234 \\
12 \%\end{array}$ & $\begin{array}{l}0.00 \\
0.61 \\
1.18 \\
2.76 \\
17 \%\end{array}$ & $\begin{array}{c}- \\
101.4 \\
80.4 \\
117.9 \\
3^{\circ}\end{array}$ & $\begin{array}{c}0.38 \\
0.68 \\
0.67 \\
3.62 \\
3 \%\end{array}$ & $\begin{array}{l}1.00 \\
1.00 \\
1.00 \\
1.00\end{array}$ & $\begin{array}{l}- \\
- \\
- \\
-\end{array}$ & 0.5 \\
\hline $\begin{array}{c}2001.17 \\
\text { VLBA } \\
15 \mathrm{GHz} \\
\\
\text { Errors }\end{array}$ & $\begin{array}{l}\mathrm{C} 1 \\
\mathrm{C} 2 \\
\mathrm{C} 3 \\
\mathrm{C} 5\end{array}$ & $\begin{array}{c}0.024 \\
1.800 \\
0.764 \\
0.179 \\
7 \%\end{array}$ & $\begin{array}{l}0.00 \\
0.35 \\
1.31 \\
4.21 \\
15 \%\end{array}$ & $\begin{array}{c}- \\
138.3 \\
94.3 \\
121.3 \\
3^{\circ}\end{array}$ & $\begin{array}{c}0.17 \\
0.39 \\
0.83 \\
4.14 \\
3 \%\end{array}$ & $\begin{array}{c}1.00 \\
0.80 \\
0.69 \\
1.00 \\
4 \%\end{array}$ & $\begin{array}{c}- \\
38.2 \\
47.0 \\
- \\
5^{\circ}\end{array}$ & 3.5 \\
\hline $\begin{array}{c}2001.98 \\
\text { VLBA } \\
15 \mathrm{GHz} \\
\text { Errors }\end{array}$ & $\begin{array}{l}\mathrm{C} 1 \\
\mathrm{C} 2 \\
\mathrm{C} 3 \\
\mathrm{C} 4\end{array}$ & $\begin{array}{c}0.091 \\
1.581 \\
0.632 \\
0.172 \\
5 \%\end{array}$ & $\begin{array}{l}0.00 \\
0.37 \\
1.42 \\
3.06 \\
10 \%\end{array}$ & $\begin{array}{c}- \\
137.9 \\
102.9 \\
112.7 \\
3^{\circ}\end{array}$ & $\begin{array}{c}0.20 \\
0.45 \\
0.77 \\
3.66 \\
6 \%\end{array}$ & $\begin{array}{c}1.00 \\
0.70 \\
0.74 \\
1.00 \\
4 \%\end{array}$ & $\begin{array}{c}- \\
36.0 \\
27.2 \\
- \\
4^{\circ}\end{array}$ & 1.6 \\
\hline
\end{tabular}


K. É. Gabányi et al.: High frequency VLBI observations of the scatter-broadened quasar B 2005+403, Online Material p 3

Table 2. continued.

\begin{tabular}{|c|c|c|c|c|c|c|c|c|}
\hline $\begin{array}{l}\text { Epoch, array and frequency } \\
\text { (1) }\end{array}$ & $\begin{array}{l}\text { Comp. } \\
\text { (2) }\end{array}$ & $\begin{array}{l}S(\mathrm{Jy}) \\
(3)\end{array}$ & $\begin{array}{c}r \text { (mas) } \\
(4)\end{array}$ & $\begin{array}{c}\theta\left(^{\circ}\right) \\
(5)\end{array}$ & $\begin{array}{c}a \text { (mas) } \\
(6)\end{array}$ & $\begin{array}{l}b / a \\
(7)\end{array}$ & $\begin{array}{c}\phi\left(^{\circ}\right) \\
(8)\end{array}$ & $\begin{array}{l}\chi_{r}^{2} \\
(9)\end{array}$ \\
\hline 2003.04 & $\mathrm{C} 1$ & 0.188 & 0.00 & - & 0.2 & 1.0 & - & 0.3 \\
\hline VLBA & $\mathrm{C} 2$ & 1.714 & 0.37 & 118.0 & 0.40 & 0.79 & 24.2 & \\
\hline \multirow[t]{3}{*}{$15 \mathrm{GHz}$} & $\mathrm{C} 3$ & 0.516 & 1.56 & 97.9 & 0.86 & 0.81 & 29.0 & \\
\hline & $\mathrm{C} 4$ & 0.008 & 2.63 & 131.4 & 0.27 & 1.00 & - & \\
\hline & $\mathrm{C} 5$ & 0.061 & 4.59 & 121.5 & 2.23 & 1.00 & - & \\
\hline Errors & & $6 \%$ & $15 \%$ & $3^{\circ}$ & $6 \%$ & $4 \%$ & $4^{\circ}$ & \\
\hline 2003.16 & $\mathrm{C} 1$ & 0.223 & 0.00 & - & 0.34 & 0.45 & 30.3 & 1.5 \\
\hline VLBA & $\mathrm{C} 2$ & 2.000 & 0.41 & 120.6 & 0.41 & 0.79 & 25.1 & \\
\hline \multirow[t]{3}{*}{$15 \mathrm{GHz}$} & $\mathrm{C} 3$ & 0.566 & 1.60 & 99.5 & 0.85 & 0.89 & 40.7 & \\
\hline & $\mathrm{C} 4$ & 0.017 & 3.00 & 124.4 & 0.99 & 1.00 & - & \\
\hline & $\mathrm{C} 5$ & 0.034 & 4.84 & 122.0 & 1.63 & 1.00 & - & \\
\hline Errors & & $4 \%$ & $12 \%$ & $3^{\circ}$ & $4 \%$ & $5 \%$ & $4^{\circ}$ & \\
\hline 1992.44 & $\mathrm{C} 1$ & 0.337 & 0.00 & - & 0.21 & 0.63 & 88.3 & 2.5 \\
\hline EVN & $\mathrm{C} 2$ & 0.723 & 0.35 & 97.4 & 0.43 & 0.79 & 37.4 & \\
\hline \multirow[t]{2}{*}{$22 \mathrm{GHz}$} & $\mathrm{C} 3$ & 0.695 & 0.65 & 87.7 & 0.20 & 0.77 & 71.2 & \\
\hline & $\mathrm{C} 5$ & 0.217 & 2.37 & 117.3 & 2.29 & 0.10 & 86.0 & \\
\hline Errors & & $10 \%$ & $10 \%$ & $3^{\circ}$ & $10 \%$ & & $5^{\circ}$ & \\
\hline 1994.17 & $\mathrm{C} 1$ & 0.570 & 0.00 & - & 0.27 & 0.68 & -54.3 & 6.3 \\
\hline VLBA+ & $\mathrm{C} 2$ & 0.796 & 0.40 & 87.7 & 0.28 & 0.56 & 62.8 & \\
\hline$+\mathrm{VLA}+\mathrm{EVN}$ & $\mathrm{C} 3$ & 0.526 & 0.81 & 82.4 & 0.24 & 0.85 & 22.7 & \\
\hline $22 \mathrm{GHz}$ & $\mathrm{C} 5$ & 0.157 & 2.56 & 112.7 & 2.32 & 0.92 & -2.7 & \\
\hline Errors & & $18 \%$ & $12 \%$ & $3^{\circ}$ & $18 \%$ & & $4^{\circ}$ & \\
\hline 1996.73 & $\mathrm{C} 1$ & 0.434 & 0.00 & - & 0.29 & 0.65 & -53.1 & 4.1 \\
\hline $\mathrm{VLBA}+\mathrm{EB}$ & $\mathrm{C} 2$ & 0.431 & 0.42 & 102.9 & 0.27 & 0.69 & 33.1 & \\
\hline \multirow[t]{2}{*}{$22 \mathrm{GHz}$} & $\mathrm{C} 3$ & 0.667 & 0.85 & 80.9 & 0.35 & 0.91 & 53.1 & \\
\hline & $\mathrm{C} 4$ & 0.212 & 2.12 & 125.7 & 4.53 & 0.28 & -72.4 & \\
\hline Errors & & $6 \%$ & $11 \%$ & $3^{\circ}$ & $18 \%$ & & $5^{\circ}$ & \\
\hline 2003.04 & $\mathrm{C} 1$ & 0.329 & 0.00 & - & 0.20 & 1.0 & - & 0.9 \\
\hline VLBA & $\mathrm{C} 2$ & 1.339 & 0.37 & 121.0 & 0.21 & 0.77 & 27.2 & \\
\hline \multirow[t]{2}{*}{$22 \mathrm{GHz}$} & $\mathrm{C} 3$ & 0.400 & 1.52 & 98.1 & 0.78 & 0.89 & 28.2 & \\
\hline & $\mathrm{C} 4$ & 0.015 & 2.77 & 127.1 & 0.41 & 1.0 & - & \\
\hline Errors & & $6 \%$ & $15 \%$ & $3^{\circ}$ & $8 \%$ & & $3^{\circ}$ & \\
\hline 1996.73 & $\mathrm{C} 1$ & 0.343 & 0.00 & - & 0.30 & 0.57 & -36.3 & 0.6 \\
\hline $\mathrm{VLBA}+\mathrm{EB}$ & $\mathrm{C} 2$ & 0.304 & 0.46 & 102.6 & 0.23 & 0.67 & -0.2 & \\
\hline \multirow[t]{2}{*}{$43 \mathrm{GHz}$} & $\mathrm{C} 3$ & 0.442 & 0.92 & 83.0 & 0.33 & 0.84 & 36.2 & \\
\hline & $\mathrm{C} 5$ & 0.191 & 3.73 & 116.4 & 3.01 & 0.66 & 35.5 & \\
\hline Errors & & $7 \%$ & $6 \%$ & $3^{\circ}$ & $12 \%$ & & $5^{\circ}$ & \\
\hline
\end{tabular}

